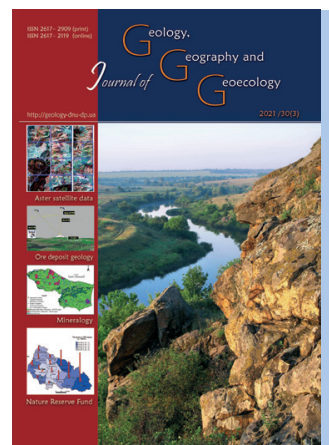

\section{Journal of Geology. Geography and Geoecology}

Journal home page: geology-dnu.dp.ua
ISSN 2617-2909 (print)

ISSN 2617-2119 (online)

Journ. Geol. Geograph. Geology, 30(3), 546-561.

doi: $10.15421 / 112150$

Mohammed R. K., Thirukumaran V., Suresh M.

Geol. Geograph. Geoecology, 30(3), 546-561

\title{
Spatial Evaluation of Groundwater Quality Using GIS and WQI in Gadilam River Basin, Tamil Nadu, India
}

\author{
K. Mohammed Rizwan ${ }^{1}$, V. Thirukumaran ${ }^{1}$, M. Suresh ${ }^{2}$ \\ ${ }^{1}$ Government Arts College (Auto), Salem-636007, India \\ 2Jayalakshmi Institute of Technology, Dharmapuri Dist., India, e-mail: mailkumaran75@gmail.com
}

Received: 17.10 .2020

Received in revised form: 13.03 .2021

Accepted: 27.04.2021

Abstract. The aims of the current research are to assess the drinking water quality of the groundwater in the Gadilam River Basin, which is located in the northern part of Tamil Nadu, by identifying the groundwater quality index and examine its suitability for drinking.

The current work determines the levels of groundwater quality parameters based on 120 groundwater samples; 50 samples from Archaean formation, 34 samples from Quaternary formation, 35 samples from Tertiary formation and the remaining sample from Cretaceous formation. Additionally, this research compares the determined levels with the various standards for drinking. Furthermore, the variability of parameters of the groundwater quality is explored in this paper by using the spatial interpolation method. The conclusion of this research reveals that the groundwater quality parameters such as $\mathrm{Calcium}\left(\mathrm{Ca}^{2+}\right)$, Magnesium $\left(\mathrm{Mg}^{2+}\right)$, Nitrate $\left(\mathrm{NO}_{3}{ }^{2-}\right)$, Fluoride $\left(\mathrm{F}^{-}\right)$, Sulphate $\left(\mathrm{SO}_{4}^{2-}\right)$, Bi-carbonate $\left(\mathrm{HCO}_{3}^{-}\right)$and Percentage of Hydrogen $(\mathrm{pH})$ values are observed to be within the limiting value for WHO 2017 in all the formations during the seasons in which they were taken. The water quality index (WQI) values of the Archaean, Quaternary and Tertiary formations are found to be less than 100 meq/L in all stations in both seasons. In order of WQI, these stations come under the category of "Excellent" and "Good". The Piper trilinear classification of groundwater samples fall in the field of mixed $\mathrm{Ca}-\mathrm{Mg}-\mathrm{Cl}$, and No dominance, some of the samples represent $\mathrm{Na}-\mathrm{K}, \mathrm{Cl}$ types of water. Keywords: Archaean, Quaternary, Tertiary, Cretaceous, groundwater, water quality.

\section{Просторова оцінка якості підземних вод з використанням геоінформаційних систем (ГІС) та індексу якості води (WQI) у басейні річки Гаділам, Тамілнад, Індія}

\author{
К. Мухаммед Різван ${ }^{1}$, В. Тірукумаран ${ }^{1}$, М. Суреш ${ }^{2}$ \\ ${ }^{1}$ Урядовий коледж мистецтв (авто), Салем-636007, Індія \\ ${ }^{2}$ Технологічний інститут Джаялакшмі, Дхармапурі, Індія, e-mail: mailkumaran75@gmail.com
}

\begin{abstract}
Анотація. Метою данного дослідження є оцінка якості підземних вод у басейні річки Гаділам, розташованої в північній частині Тамілнаду як джерела питної води, шляхом визначення індексу якості підземних вод та вивчення їх придатності для питного споживання. Представлена робота визначає рівні параметрів якості підземних вод на підставі дослідження 120 проб підземних вод: 50 зразків з архейської формації, 34 зразка з четвертинної формації, 35 зразків з третинної формації та однієї проби з крейдової формації. Крім того, це дослідження порівнює визначені рівні з різними стандартами до питної води. Крім того, мінливість параметрів якості підземних вод досліджується в цій роботі за допомогою методу просторової інтерполяції. На завершення цього дослідження виявляється, що такі параметри якості підземних вод, як кальцій $\left(\mathrm{Ca}^{2+}\right)$, магній $\left(\mathrm{Mg}^{2+}\right)$, нітрат $\left(\mathrm{NO}_{3}^{2-}\right)$, фтор ( $\left.\mathrm{F}^{-}\right)$, сульфат $\left(\mathrm{SO}_{4}^{2-}\right)$, бікарбонат $\left(\mathrm{HCO}^{3-}\right)$ та водневий показник $(\mathrm{pH})$ спостерігаються в межах граничного значення для ВООЗ 2017 у всіх формаціях протягом цього сезону. Значення індексу якості води архейських, четвертинних та третинних утворень (WQI) є меншими за 100 мекв/л на всіх станціях в обидва сезони. Відповідно оцінки WQI, ці пункти спостереження належать до категорії «Відмінно» та «Добре». За трилінійною класифікацією Пайпера проби підземних вод потрапляють в область змішаних $\mathrm{Ca}-\mathrm{Mg}-\mathrm{Cl}$ із відсутнім домінуванням, деякі зразки у типах $\mathrm{Na}-\mathrm{K} \mathrm{i} \mathrm{Cl}$ типи води.
\end{abstract}

Ключові слова: архей, четвертинний, третинний, крейдовий період, підземні води, якість води.

\section{Introduction.}

Water plays a vital role in human life. The consequences of urbanization and industrialization lead to deterioration of the quality of the water (Sanjay et al 2007). During the last few decades, it has been observed that groundwater is becoming polluted drastically because of rapid industrialization, improper dumping of solid wastes and toxic waste management (Yousefi et al., 2018). Consequently, the number of cases of waterborne diseases causes health hazards, while the use of such water for agricultural purposes lead to adverse effects on crop production and fertility 
of agricultural soils (Pirsaheb et al., 2014; Asghari et al., 2018; Muthusaravanan et al., 2018).

All over the world, groundwater is used for many purposes including irrigation, domestic, moreover industrial uses. Pollution has increased in the last few decades due to the continuous-increment in population. This increase is significant to the demand for fresh water as a result of the rapid development of the population and the expanded rate of progress in industrialization. Decrease in water quality happens to be a worldwide issue of focus since human populations are increasing, agricultural and industrial activities are expanding, additionally climate change poses the threat of major changes to the hydrological-cycle (Federation and APHA, 2005).

The groundwater quality depends mainly on the geological formations and anthropogenic activities. There are many studies regarding the excessive extraction of groundwater and resulting sea water intrusion contaminating coastal aquifers (Bagade, 1995; Thigale et al., 1998a; Duraiswami et al., 2000). Increased usage of groundwater has depleted the sources of groundwater. The excess concentration of certain ions has made the water unfit for drinking and irrigation uses. The discharges from industries and land resources have led to serious problems in the water quality. Public health and agriculture have been gravely impaired due to consumption of contaminated water and depleted groundwater source.

According to the WHO organization (WHO, 2017), nearly $80 \%$ of all human diseases are caused by water. Hence the quality of water must be expressed in the most common form to analyze the water characteristics. Whenever groundwater is contaminated or degraded, its quality fails to recover unless contaminants are prevented from penetrating through the source. The guidelines and standards for drinking water quality are planned to ensure that perfectly clean and protected water is distributed for human consumption, with the intention of protecting people's health. For that reason, it is necessary to continuously monitor the groundwater quality and to protect it.

The general target of any assessment for groundwater quality is often to get an all-inclusive evaluation of variations of the quality of groundwater and evaluate the changes that occur in the groundwater quality over time, either in a natural way or under human pressure (Tiwari and Nayak, 2002).

Several authors have studied the hydrogeochemistry of groundwater and vulnerability of the aquifers to pollution in hard rock aquifer of peninsular India. Rina et al., (2011), Singh et al., (2012a and 2012b) studied hydrogeochemical evolution of groundwater. Prasanna et al., (2011), Sonkamble et al., (2012), Brindha et al., (2013), Brindha and Kavitha (2014), Kumar et al., (2014), Rajesh et al., (2015) investigated aquifers in various rock domains like granite, gneiss, schist and basalt to enumerate the geochemical evolution of groundwater and its suitability for various use.

The Water Quality Index (WQI) is the most considerable tool that is effective in conveying information about water quality to concerned citizens along with policy makers. It really is a strategy which is efficient in determining the water characteristics (Singh 1992, Naik et al. 2001, Mishra et al. 2001). The Water Quality Index therefore, becomes a crucial parameter used in the management and assessment of groundwater. It can help in classifying groundwater: whether or not it is fit for irrigation. WQI is computed from the standpoint of groundwater suitability for irrigation consumption. WQI is distinctive as a score that reflects the composite-effect of a variety of water quality parameters. The calculation of the WQI in groundwater study started with Horton (1965) and Landwehr (1974). Wu et al. (2011) reported that the selection of water quality parameters is a necessity in evaluating the primary anthropogenic activity in the monitoring location. The primary anthropogenic activity may be domestic, agriculture, mining, etc. It is possible to determine the groundwater quality index (GWQI) by analyzing several important parameters and assign a proper weight for each one.

\section{Study Area.}

The Gadilam River originates from Kallakurichi district and flows through Viluppuram district to meet the Bay of Bengal in Cuddalore district. This river total flow length is $102 \mathrm{~km}$ and catchment area is 2091.20 sq.km. It is reported that the river gets a supply of occasional floodwater from the Ponnaiyar River through the Malattat River. The Gadilam River basin is extended from $11^{\circ} 26^{\prime} 31.797^{\prime \prime} \mathrm{N}$ to $11^{\circ} 56^{\prime} 29.633^{\prime \prime} \mathrm{N}$ latitudes and 78 59'10.675” E to 7947'15.793” E longitudes (Fig. 1).

It is represented on Survey of India topographical maps $58 \mathrm{I} / 13,58 \mathrm{M} / 1,58 \mathrm{M} / 2,58 \mathrm{M} / 5,58 \mathrm{M} / 6,58 \mathrm{M} / 7$, $58 \mathrm{M} / 9,58 \mathrm{M} / 10,58 \mathrm{M} / 11$ and $58 \mathrm{M} / 15$ on a scale of $1: 50,000$. The study area is bounded by Villupuram in the north, Cuddalore town in the east, Thirukoilur in west, and Vadalur in the south. There are a number (1024) of tanks in the study area, of which, 62 major (above 0.5 sq. $\mathrm{km}$ ) tanks are noted. The temperature reaches its maximum during April and May $38^{\circ}$ to $39^{\circ} \mathrm{C}$, minimum in January and February $24^{\circ}$ to $25^{\circ} \mathrm{C}$. The wind velocity is highest during summer and sometimes during the monsoon seasons.

The present study area catchment area of the basin is located in Kallakurichi district, the mature stage of the river basin flow in Viluppuram district and the lower river basin is in Cuddalore district. Agriculture is the major occupation in the entire Gadilam River 

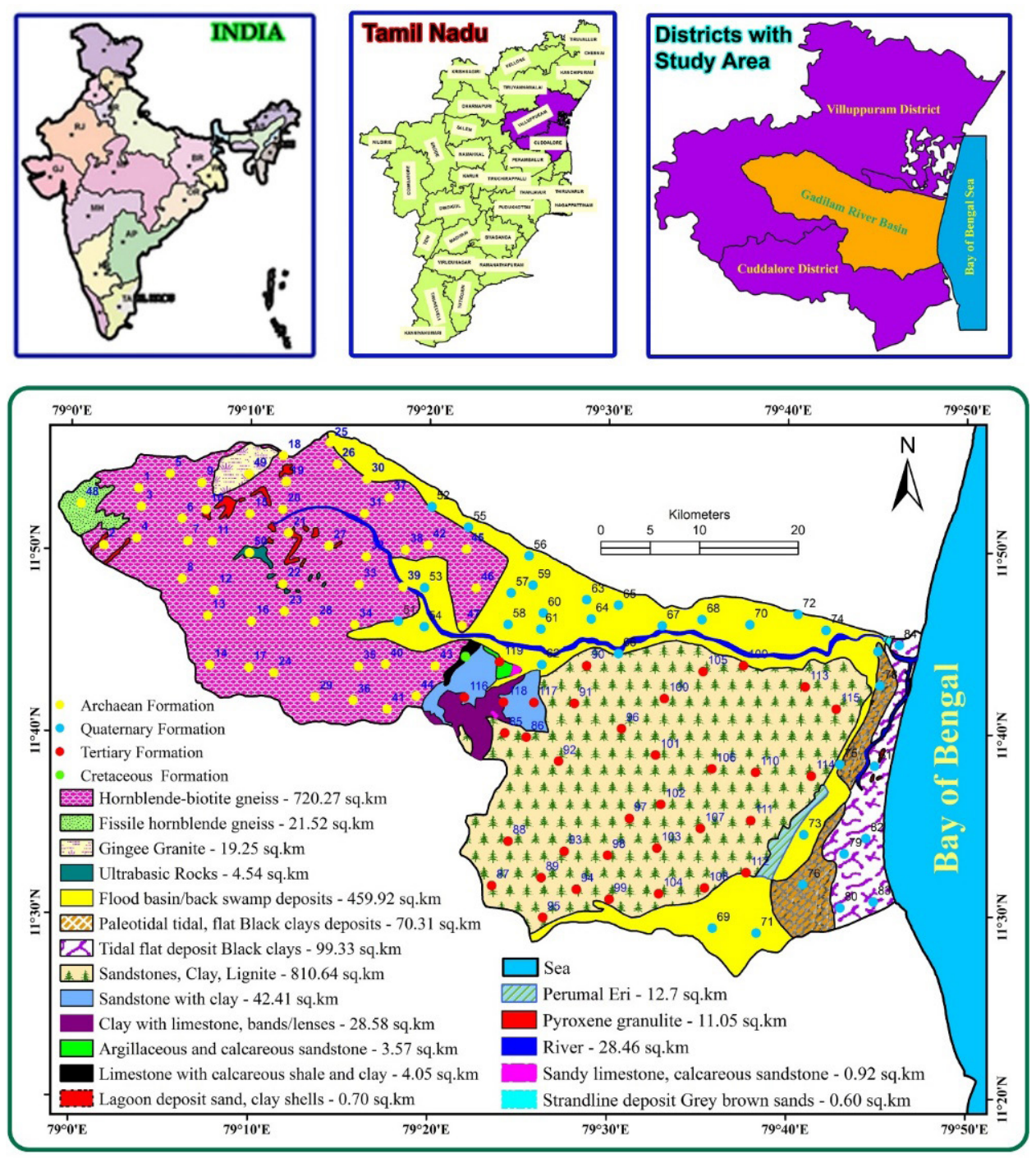

Fig. 1. Key Map of Study Area

Basin. Most of the area's irrigational, domestic and drinking needs are supplied by groundwater resources, and demand for this has increased in recent times. The drilling of new bore wells and extensive exploitation of groundwater have affected the groundwater quality of the study area. Besides, large scale farming and paddy cultivation leads to use powerful pumps for withdrawing groundwater, and this could deplete the sources at a much faster rate. Hence, an understanding of groundwater quality characteristics for drinking and irrigational needs of the region is necessary in order to ensure continuous agricultural activity. Besides, the study of groundwater quality characteristics has not been attempted in the recent times in the study area (Shankar et al., 2011; Layeek Ahamed et al., 2014).

\section{Materials and Methods.}

The Gadilam River Basin boundary is demarcated based on the drainage system using Survey of India topographical maps $58 \mathrm{I} / 13,58 \mathrm{M} / 1,58 \mathrm{M} / 2,58 \mathrm{M} / 5$, $58 \mathrm{M} / 6,58 \mathrm{M} / 7,58 \mathrm{M} / 9,58 \mathrm{M} / 10,58 \mathrm{M} / 11$ and $58 \mathrm{M} / 15$ on 1:50,000 scale and drainage updating in current satellite data (Landsat-8 TM data, March-2018). The geology map is traced from the district resources map. The Gadilam River upper basin shows an Archaean formation and the lower basin shows Tertiary uplands in the south and recent alluvium (Quaternary) in the north (Fig. 1). A detailed methodology flowchart is given in Fig. 2.

Overall, 120 groundwater samples were collected from the Gadilam River Basin excluding the reserved forest area. Fig. 1 shows the distribution of the 50 


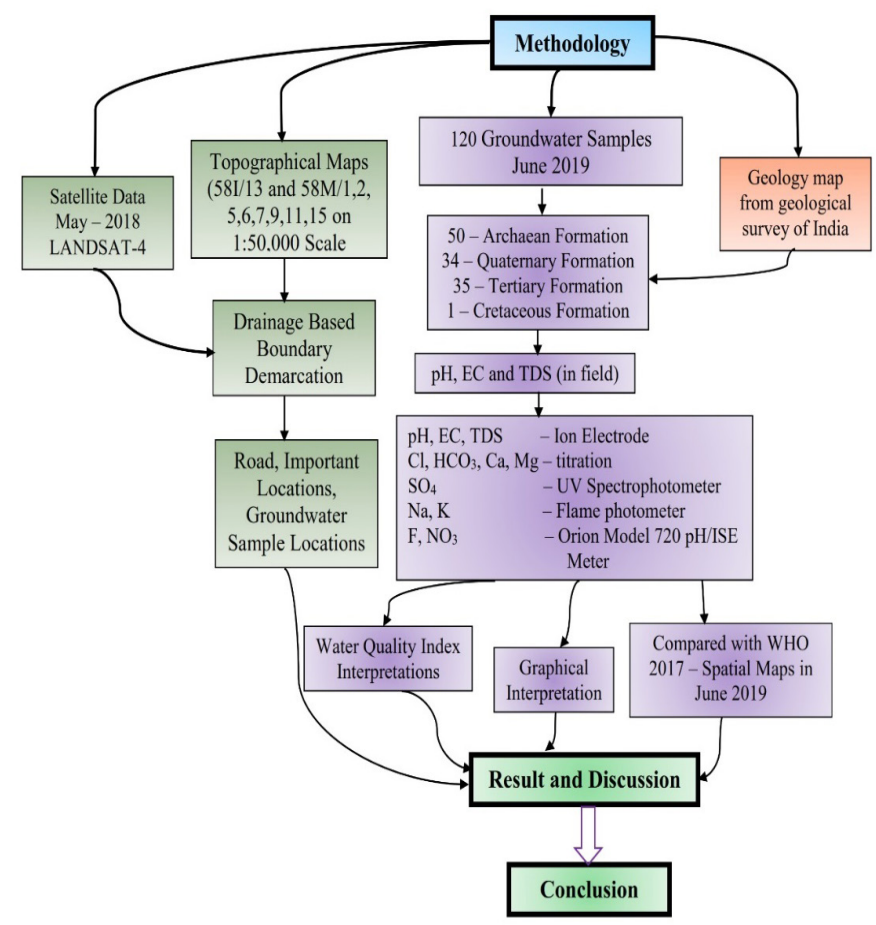

Fig. 2. Detailed methodology flowchart

samples which were collected from the Archaean formation (hornblende-biotite gneiss, fissile hornblende gneiss, Gingee granite and ultrabasic Rocks). 34 samples from the Quaternary formation (flood basin/back swamp deposits, Paleotidal tidal, flat black clay deposits, tidal flat deposit black clays) and another 35 samples from Tertiary formation (sandstones, clay, lignite, sandstone with clay, argillaceous). The single remaining sample is from the Cretaceous formation. Groundwater samples were collected using 1 liter plastic containers. To ensure the collection of representative groundwater samples from the borehole and dug-wells groundwater samples were collected during June 2019. The analysis of elements and parameters in the laboratory followed the standard methods. Each water sample from the collected samples was assessed for fourteen parameters such as TDS, TH, pH, EC, chloride, sulphate, sodium, magnesium, calcium, nitrate, potassium, fluoride and bi-carbonate using standard-procedures of water test advised by the Federation and APHA (2005).

At each location the coordinates were taken using a GPS, GARMIN eTrex 10 model. These locations were entered into GIS software and we developed the attribute in points with spatial data. The spatial distribution maps of $\mathrm{pH}, \mathrm{EC}, \mathrm{TDS}$ and major cations, anions and selected heavy metal were produced using Arc GIS 10.2 using the inverse distance weighted method IDW (Suresh et al., 2010; Vetrimurugan et al., 2017).

Each and every parameter was correlated with WHO 2017 and BIS 2012 standards. Any measurement above the value of the same standards was assigned a classified risk hazard rank. Based on this rank, spatial thematic maps were prepared. After creating the entire thematic layers using SMCE, overlay analysis was conducted using all the thematic layers and finally, to identify the risk hazard and high risk hazard zones.

Groundwater quality index (GWQI) is calculated in accordance with the following equation.

$$
G W Q I=\frac{\sum_{i=1}^{n} W_{1} q_{1}}{\sum_{i=1}^{n} W_{1}}
$$

The quality rating is calculated according to the following equation.

$$
q_{n 1}=\frac{\left(V_{\text {actual }} V_{\text {ideal }}\right)}{\left(V_{\text {standard }} V_{\text {ideal }}\right)} \times 100
$$

where,

$\mathrm{q}_{\mathrm{ni}}$ is the quality rating of the $\mathrm{i}^{\text {th }}$ parameter for the total (n) number of the water quality parameters.

$\mathrm{V}_{\text {actual }}$ is the measured value of water quality parameter (found from the laboratory).

$\mathrm{V}_{\text {ideal }}$ is the standard value of water quality parameter (found from standard tables).

The value of $\mathrm{V}_{\text {ideal }}$ for $\mathrm{pH}$ is 7 and for the other studied water quality parameters is zero.

\section{Results and Discussion.}

The Archaean, Quaternary, Tertiary formation minimum, maximum, average and standard deviation values of physio-chemical parameters during November 2018 results are shown in Table 1. 


\begin{tabular}{|c|c|c|c|c|c|c|c|c|c|c|c|c|c|c|c|c|}
\hline \multirow{4}{*}{ 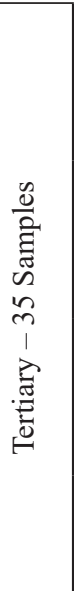 } & \multirow{12}{*}{ 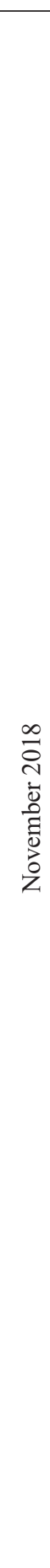 } & 岕 & $\begin{array}{l}\infty \\
\stackrel{\text { ते }}{\text { הे }}\end{array}$ & $\begin{array}{l}\infty \\
\stackrel{\infty}{=} \\
=\end{array}$ & $\begin{array}{l}\dot{\theta} \\
\ddot{\gamma}\end{array}$ & $\stackrel{t}{n}$ & $\begin{array}{l}\stackrel{0}{0} \\
\dot{+}\end{array}$ & $\begin{array}{l}\vec{\infty} \\
\stackrel{j}{ }\end{array}$ & ते & $\begin{array}{l}\dot{J} \\
\dot{J} \\
\dot{0}\end{array}$ & 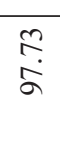 & 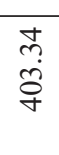 & กุ & 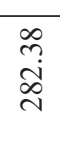 & $\begin{array}{l}\hat{\hat{\sigma}} \\
\hat{\sigma}\end{array}$ & 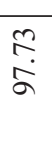 \\
\hline & & 安 & $\begin{array}{l}\infty \\
\infty \\
\delta \\
\delta\end{array}$ & $\begin{array}{l}\stackrel{\tilde{N}}{\mathrm{~d}} \\
\stackrel{\mathrm{d}}{ }\end{array}$ & $\begin{array}{l}\stackrel{2}{\infty} \\
\infty \\
\infty\end{array}$ & $\begin{array}{l}\text { I } \\
\text { İ }\end{array}$ & $\stackrel{t}{\stackrel{a}{a}}$ & 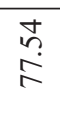 & m. & $\begin{array}{l}\stackrel{0}{1} \\
\text { in } \\
=\end{array}$ & $\begin{array}{l}\hat{\sigma} \\
\mathrm{I}\end{array}$ & $\begin{array}{l}\hat{n} \\
\hat{o} \\
\infty\end{array}$ & $\stackrel{n}{n}$ & $\begin{array}{l}\hat{i} \\
\stackrel{0}{0} \\
i\end{array}$ & $\begin{array}{l}\bar{n} \\
\stackrel{\circ}{\sim}\end{array}$ & 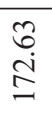 \\
\hline & & 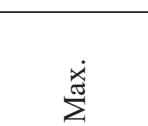 & $\begin{array}{l}\stackrel{8}{0} \\
\stackrel{0}{-1}\end{array}$ & $\begin{array}{l}8 \\
8 \\
8\end{array}$ & $\begin{array}{l}8 \\
\stackrel{0}{\circ} \\
0\end{array}$ & $\begin{array}{l}8 \\
\dot{+} \\
\dot{+}\end{array}$ & $\begin{array}{l}8 \\
\stackrel{i}{+}\end{array}$ & $\begin{array}{l}8 \\
\stackrel{8}{+} \\
\stackrel{+}{N}\end{array}$ & 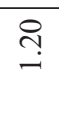 & $\begin{array}{l}8 \\
0 \\
0 \\
0\end{array}$ & $\begin{array}{l}\text { \& } \\
\text { ర్ల }\end{array}$ & $\begin{array}{l}\stackrel{8}{.} \\
\infty \\
\infty\end{array}$ & $\vec{\infty}$ & $\underset{\sim}{\stackrel{8}{\pi}}$ & $\begin{array}{l}8 \\
0 \\
o \\
0\end{array}$ & $\begin{array}{l}\text { \&े } \\
\text { id } \\
\text { in }\end{array}$ \\
\hline & & $\dot{\Xi}$ & $\underset{0}{8}$ & $\underset{1}{\circ}$ & $\begin{array}{l}8 \\
\stackrel{2}{0} \\
\text { in }\end{array}$ & $\underset{+}{\stackrel{8}{+}}$ & $\underset{r}{8}$ & $\begin{array}{l}\stackrel{8}{8} \\
\stackrel{\sim}{+}\end{array}$ & 8 & $\begin{array}{l}8 \\
\dot{+}\end{array}$ & $\begin{array}{l}8 \\
\text { ¿े } \\
\text { in }\end{array}$ & $\begin{array}{l}8 \\
\infty \\
\infty \\
\infty \\
\text { N }\end{array}$ & $\vec{ָ}$ & $\begin{array}{l}\text { \& } \\
\text { iิ }\end{array}$ & $\begin{array}{l}8 \\
i \\
i\end{array}$ & $\begin{array}{l}8 \\
\text { : } \\
\text { in }\end{array}$ \\
\hline \multirow{4}{*}{ 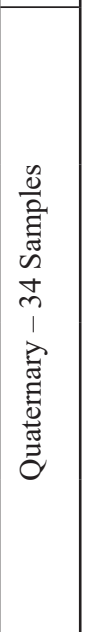 } & & $\begin{array}{l}\overrightarrow{\grave{D}} \\
\dot{\vec{D}} \\
\dot{n}\end{array}$ & $\begin{array}{l}\stackrel{a}{+} \\
\infty \\
i\end{array}$ & $\begin{array}{l}0 \\
\stackrel{\sigma}{0} \\
\varrho\end{array}$ & $\begin{array}{l}\text { đ̃ } \\
\stackrel{\Xi}{\Xi}\end{array}$ & 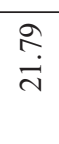 & $\overbrace{\substack{\infty \\
.}}^{\infty}$ & $\begin{array}{l}\hat{n} \\
\infty \\
\infty\end{array}$ & $\hat{\tilde{n}}$ & $\begin{array}{l}+ \\
\stackrel{0}{0}\end{array}$ & $\begin{array}{l}\infty \\
\stackrel{+}{+} \\
=\end{array}$ & $\begin{array}{l}\text { : } \\
: \\
\stackrel{+}{1}\end{array}$ & กิ & $\begin{array}{l}8 \\
\infty \\
\infty \\
\vec{n}\end{array}$ & $\begin{array}{l}\vec{J} \\
\stackrel{0}{a}\end{array}$ & $\begin{array}{l}\stackrel{\infty}{+} \\
\stackrel{+}{-}\end{array}$ \\
\hline & & 定 & $\begin{array}{l}\overrightarrow{7} \\
\dot{8}\end{array}$ & $\begin{array}{l}\infty \\
\infty \\
m \\
m\end{array}$ & $\begin{array}{l}8 \\
\stackrel{1}{1}\end{array}$ & $\begin{array}{l}\stackrel{\cap}{n} \\
\stackrel{+}{\sim}\end{array}$ & $\begin{array}{l}\tilde{\sigma} \\
\stackrel{2}{-}\end{array}$ & $\begin{array}{l}\vec{R} \\
\stackrel{0}{\Xi} \\
=\end{array}$ & fo & $\begin{array}{l}\text { N̦ } \\
\text { in }\end{array}$ & $\begin{array}{l}\infty \\
\infty \\
0 \\
0 \\
0\end{array}$ & 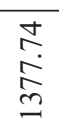 & $\underset{\sim}{\stackrel{+}{r}}$ & $\begin{array}{l}\frac{n}{+} \\
\dot{d} \\
a\end{array}$ & $\begin{array}{l}\stackrel{8}{0} \\
\dot{0}\end{array}$ & $\begin{array}{l}\infty \\
\infty \\
0 \\
\stackrel{0}{0} \\
\text { N. }\end{array}$ \\
\hline & & $\stackrel{\dot{x}}{\dot{x}^{\oplus}}$ & $\begin{array}{l}8 \\
\dot{9} \\
\end{array}$ & $\begin{array}{l}8 \\
\dot{\infty}\end{array}$ & $\begin{array}{l}8 \\
\dot{8} \\
8\end{array}$ & $\begin{array}{l}8 \\
\stackrel{8}{0}\end{array}$ & $\begin{array}{l}8 \\
\stackrel{\text { ते }}{ }\end{array}$ & $\begin{array}{l}\underset{\infty}{\infty} \\
\stackrel{\infty}{\infty}\end{array}$ & 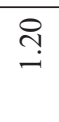 & $\begin{array}{l}8 \\
\dot{+} \\
\text { ले }\end{array}$ & $\begin{array}{l}8 \\
\dot{+} \\
\text { ర }\end{array}$ & $\begin{array}{l}8 \\
\dot{8} \\
\dot{8} \\
\dot{0}\end{array}$ & $\begin{array}{l}n \\
\infty \\
\infty\end{array}$ & $\begin{array}{l}8 \\
\dot{0} \\
\infty \\
\infty\end{array}$ & \begin{tabular}{l}
8 \\
$\dot{0}$ \\
\multirow{2}{*}{}
\end{tabular} & $\begin{array}{l}8 \\
\dot{\Xi} \\
\text { ปे }\end{array}$ \\
\hline & & $\dot{\Xi}$ & $\begin{array}{l}\dot{8} \\
\dot{\lambda}\end{array}$ & 8 & $\begin{array}{l}8 \\
\ddot{n} \\
m\end{array}$ & $\underset{\text { in }}{8}$ & $\underset{j}{\stackrel{8}{ }}$ & $\begin{array}{l}\stackrel{8}{8} \\
\dot{\sim}\end{array}$ & 8 & $\begin{array}{l}8 \\
\dot{+}\end{array}$ & $\begin{array}{l}8 \\
8 \\
i n\end{array}$ & $\underset{\substack{i \\
\text { i }}}{8}$ & $\stackrel{\Xi}{\cong}$ & $\begin{array}{l}8 \\
8 \\
0 \\
0\end{array}$ & $\begin{array}{l}8 \\
\infty \\
\infty \\
\infty\end{array}$ & $\begin{array}{l}8 \\
\text { i. } \\
\text { in }\end{array}$ \\
\hline \multirow{4}{*}{ 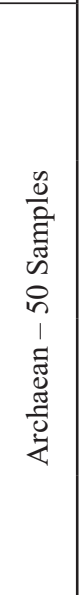 } & & $\begin{array}{l}\overrightarrow{\mathrm{D}} \\
\stackrel{0}{\overrightarrow{0}}\end{array}$ & $\begin{array}{l}\stackrel{n}{n} \\
\stackrel{n}{N}\end{array}$ & $\begin{array}{l}\text { f } \\
\ddot{n}\end{array}$ & $\begin{array}{l}\stackrel{ }{\longrightarrow} \\
\vec{r}\end{array}$ & $\stackrel{\infty}{\stackrel{\infty}{a}}$ & $\underset{\sim}{\mathbb{H}}$ & $\begin{array}{l}\hat{b} \\
\dot{a}\end{array}$ & iे & 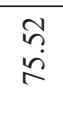 & $\begin{array}{l}\hat{a} \\
\hat{\imath}\end{array}$ & $\begin{array}{l}m \\
\dot{b} \\
\dot{b}\end{array}$ & 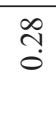 & 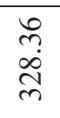 & $\begin{array}{l}\hat{\mathrm{a}} \\
\hat{\mathrm{d}}\end{array}$ & बे \\
\hline & & 安 & $\begin{array}{l}\stackrel{0}{+} \\
\stackrel{\infty}{0}\end{array}$ & $\begin{array}{l}0 \\
\stackrel{\sim}{i} \\
\stackrel{f}{f}\end{array}$ & $\begin{array}{l}\stackrel{J}{+} \\
\stackrel{f}{\exists}\end{array}$ & $\underset{\stackrel{\sim}{\sim}}{\stackrel{d}{d}}$ & $\begin{array}{l}\circ \\
\stackrel{2}{े} \\
\stackrel{1}{2}\end{array}$ & 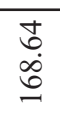 & $\begin{array}{l}\infty \\
n \\
0\end{array}$ & 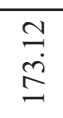 & $\begin{array}{l}\text { I } \\
\stackrel{0}{0} \\
\infty \\
\text { N }\end{array}$ & $\begin{array}{l}\stackrel{P}{P} \\
\dot{\infty} \\
\stackrel{+}{ \pm}\end{array}$ & $\bar{n}$ & $\begin{array}{l}\dot{J} \\
\dot{ \pm} \\
\end{array}$ & 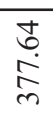 & $\begin{array}{l}+ \\
0 \\
\dot{0} \\
0 \\
N\end{array}$ \\
\hline & & 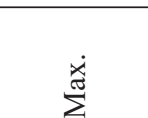 & $\begin{array}{l}8 \\
0 \\
0 \\
\end{array}$ & $\underset{8}{\stackrel{8}{1}}$ & $\begin{array}{l}8 \\
\stackrel{8}{0} \\
\text { in }\end{array}$ & $\begin{array}{l}8 \\
\text { in }\end{array}$ & $\begin{array}{l}8 \\
\dot{q}\end{array}$ & $\begin{array}{l}8 \\
\dot{\infty} \\
\stackrel{\infty}{+}\end{array}$ & ભิ & $\begin{array}{l}8 \\
\text { ¿ } \\
\text { d }\end{array}$ & \begin{tabular}{l}
8 \\
$\dot{0}$ \\
\multirow{7}{*}{}
\end{tabular} & $\begin{array}{l}8 \\
8 \\
8 \\
8 \\
0\end{array}$ & $\underset{\infty}{\mathcal{C}}$ & $\begin{array}{l}8 \\
8 \\
\dot{\Pi} \\
\infty\end{array}$ & $\begin{array}{l}8 \\
\\
\dot{0} \\
0\end{array}$ & 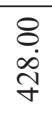 \\
\hline & & $\dot{\Xi}$ & $\begin{array}{l}8 \\
\stackrel{\Delta}{\sim} \\
\end{array}$ & $\begin{array}{l}\stackrel{8}{ } \\
\text { i }\end{array}$ & $\begin{array}{l}8 \\
\dot{+} \\
\dot{+}\end{array}$ & 8 & $\begin{array}{l}\stackrel{8}{+} \\
\dot{+}\end{array}$ & $\begin{array}{l}8 \\
\dot{\infty} \\
\infty\end{array}$ & 8 & $\begin{array}{l}8 \\
\text { in }\end{array}$ & $\begin{array}{l}8 \\
\text { in } \\
\text { in }\end{array}$ & $\begin{array}{l}8 \\
8 \\
\dot{+} \\
y\end{array}$ & 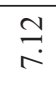 & $\begin{array}{l}8 \\
\dot{\infty} \\
\frac{\infty}{m}\end{array}$ & $\begin{array}{l}8 \\
\stackrel{0}{0} \\
=\end{array}$ & $\begin{array}{l}8 \\
\text { i } \\
\text { in }\end{array}$ \\
\hline \multirow{2}{*}{ 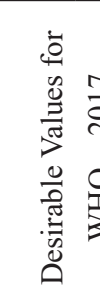 } & & 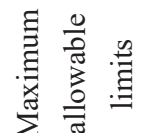 & 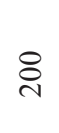 & $\stackrel{n}{n}$ & $\underset{్}{~}$ & $\simeq$ & if & 8 & $\stackrel{n}{\longrightarrow}$ & \& & \& & 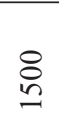 & กั & 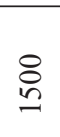 & \& & in \\
\hline & & 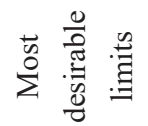 & $\curvearrowleft$ & in & ' & ' & ' & $\underset{\text { ¿ }}{8}$ & ' & $\underset{\text { ¿ }}{8}$ & ষ্লల & ' & $\begin{array}{l}n \\
\infty \\
1 \\
n \\
n \\
0\end{array}$ & \& & 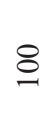 & ' \\
\hline \multicolumn{3}{|c|}{ 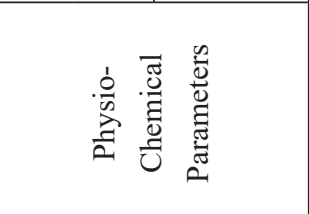 } & 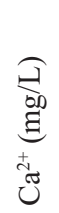 & 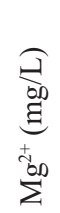 & 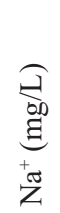 & 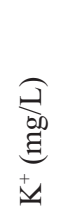 & 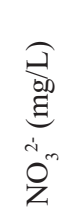 & 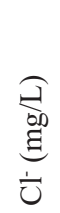 & 空 & 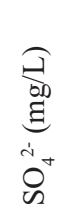 & 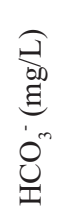 & 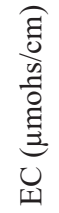 & $\stackrel{I}{2}$ & 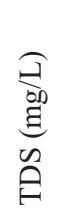 & 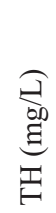 & 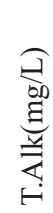 \\
\hline
\end{tabular}


The concentration of the Calcium $\left(\mathrm{Ca}^{2+}\right)$, Magnesium $\left(\mathrm{Mg}^{2+}\right)$, Nitrate $\left(\mathrm{NO}_{3}{ }^{2-}\right)$, Fluoride $\left(\mathrm{F}^{-}\right)$, Sulphate $\left(\mathrm{SO}_{4}^{2-}\right)$, Bi-carbonate $\left(\mathrm{HCO}_{3}{ }^{-}\right)$and percentage of Hydrogen $(\mathrm{pH})$ values are observed to be within the limiting value for WHO 2017 in all the formations during this season.

Sodium $\left(\mathrm{Na}^{+}\right)$values were found to be not permissible in some samples from the Quaternary and Archaean formations due to the presence of concentrated colloids in the water (Akhilesh Jinwal et al., 2008). All sample values were observed to be within the limiting value for WHO 2017 in the Tertiary formation. The sodium concentration of the three formations shows that the Quaternary formation has the highest value (600 $\mathrm{mg} / \mathrm{L}$ ) and in the Archaean formation the maximum value $(250 \mathrm{mg} / \mathrm{L})$ was noticed.

From the spatial variation with contour lines of the Na map (Fig. 3) excess over the limit for drinking purposes was seen, twelve samples in these sample places in the Archaean formation were located in between (contact zone) hornblende-biotite gneiss and the flood basin. Therefore, it is the weaker plane which may have significant influence of rock water interaction (Rajmohan et al., 2000).

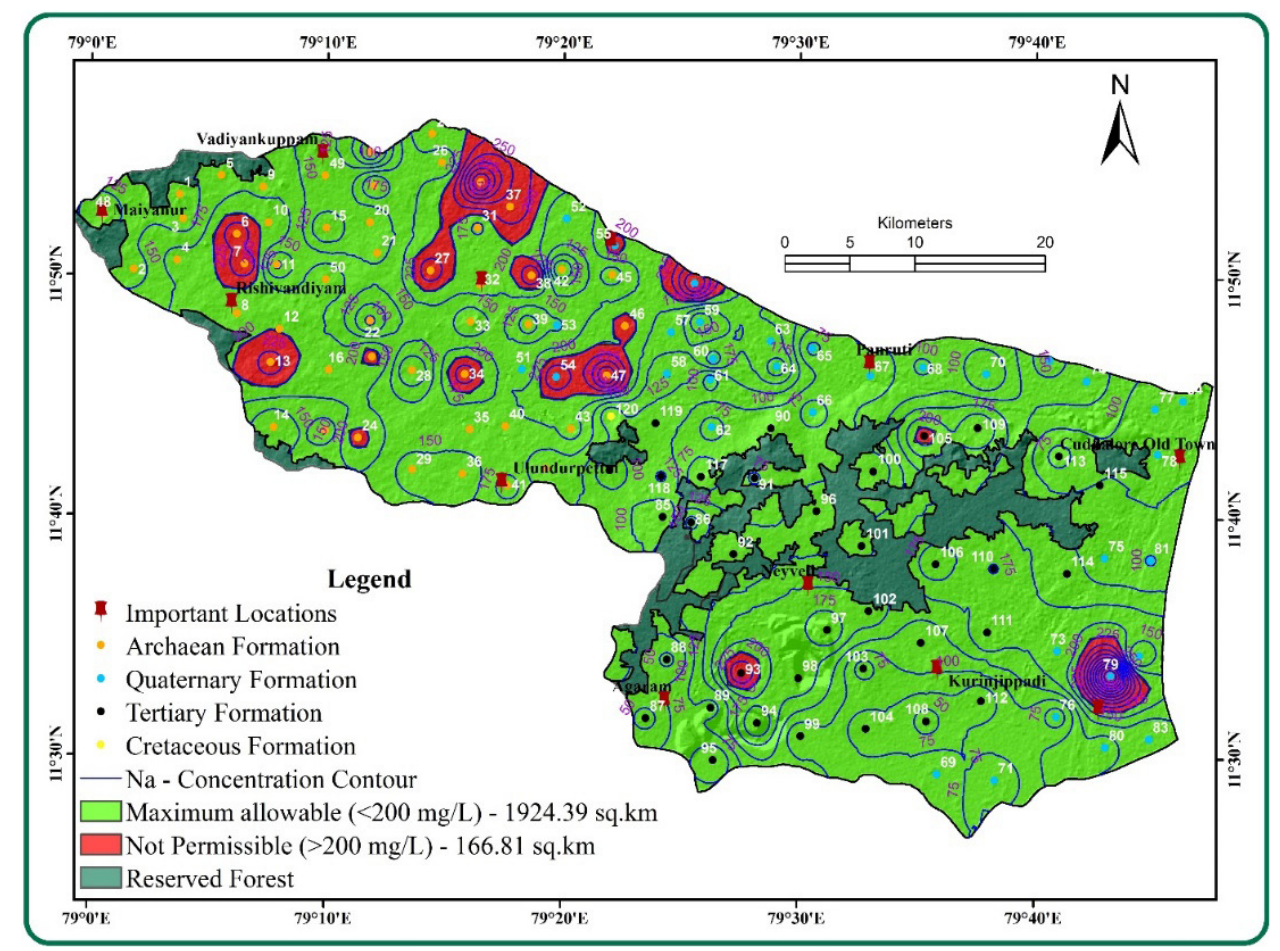

Fig. 3. Sodium spatial variation map - WHO 2017

Only four such samples were studied in the Quaternary formation. These sample places were located in between (contact zone) the flood basin deposit with hornblende-biotite gneiss and the sea shoreline. Therefore, it is the weaker plane that may have considerable influence of rock water interaction and there may be sea water intrusion to the fresh groundwater.

In the Tertiary formation only two samples of groundwater were found to be outside the permissible limit. These sample places were located in between (contact zone) sand stone, clay, lignite deposit with flood basin deposit and nearby lignite mining area. Therefore, there may be significant influence of rock water interaction and anthropogenic activities.

The highest concentration was found along the north and north western area of the catchment area and the east coast of the Gadilam River Basin. These results indicated the leaching of secondary salts on the upstream side and that there may be sea water intrusion in the downstream side of the east coast study area.

Potassium $\left(\mathrm{K}^{+}\right)$and Total Hardness (Figures 4 and 5) values are found to be in excess of the permissible values in the majority of samples in all the formations. The sequence of high concentration is as follows; Quaternary $>$ Archaean $>$ Tertiary. The sodium and Total Hardness values of the three formations show that the Quaternary formation has the highest value of $\mathrm{K}(100 \mathrm{mg} / \mathrm{L})$ and TH $(704 \mathrm{mg} / \mathrm{L})$. In the Quaternary formation $94 \%$ of the samples exceeded the limit for WHO 2017. 


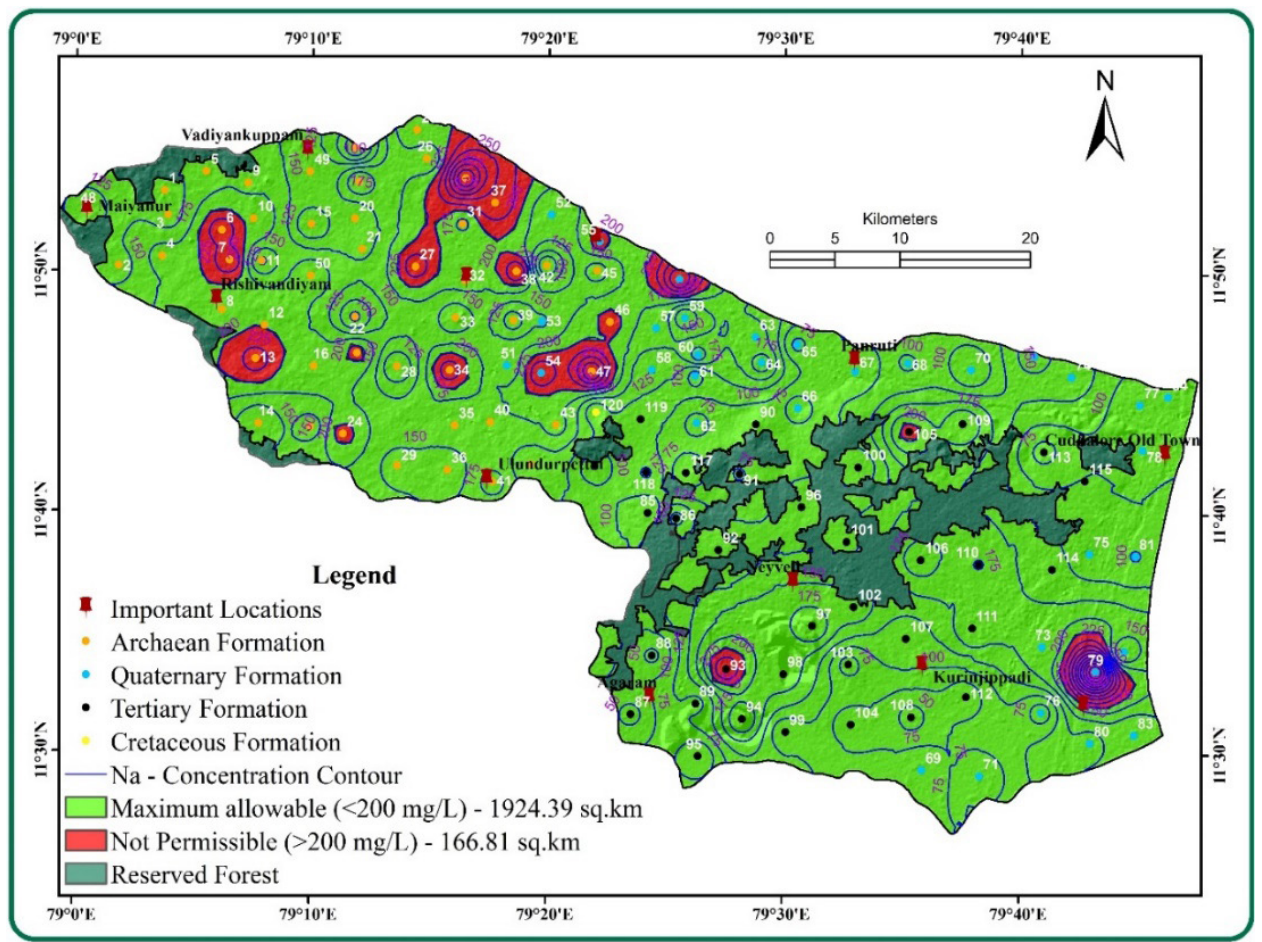

Fig. 4. Potassium spatial variation map - WHO 2017

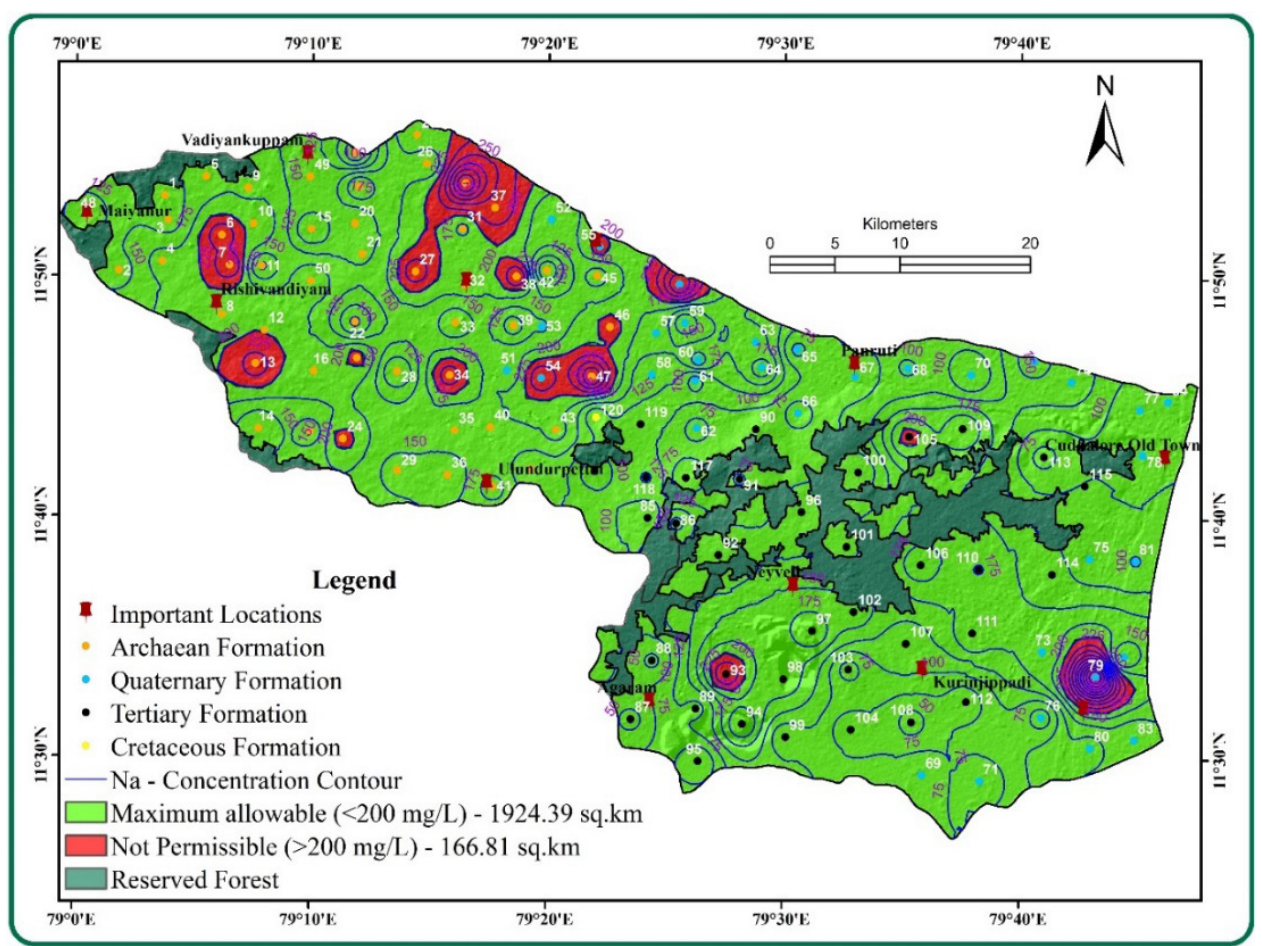

Fig. 5. Sodium spatial variation map - WHO 2017

The Archaean formation maximum value of $\mathrm{K}$ was $50 \mathrm{mg} / \mathrm{L}$ and $\mathrm{TH}$ value $(684 \mathrm{mg} / \mathrm{L})$. All the samples from the Archaean formation were observed to exceed the limit. In the Tertiary formation the highest value of $\mathrm{K}(40 \mathrm{mg} / \mathrm{L})$ and TH $568 \mathrm{mg} / \mathrm{L}$ was noticed. The Tertiary formation groundwater quality was noticed in $89 \%$ of the samples to be outside the permissible limit.High potassium values may cause nervous and digestive disorders (Ambrina Sardar Khan et al., 2012). The highest values are due to the greater depth of water level and high rate of evaporation during the hot season (Mahmoud et al., 2016).

Chloride $\left(\mathrm{Cl}^{-}\right)$and Total Alkalinity (Figures 6 and 7) values are found to be not permissible in a minimum number of samples only in the Quaternary formation. The Chloride $\left(\mathrm{Cl}^{-}\right)$and Total Alkalinity values show 


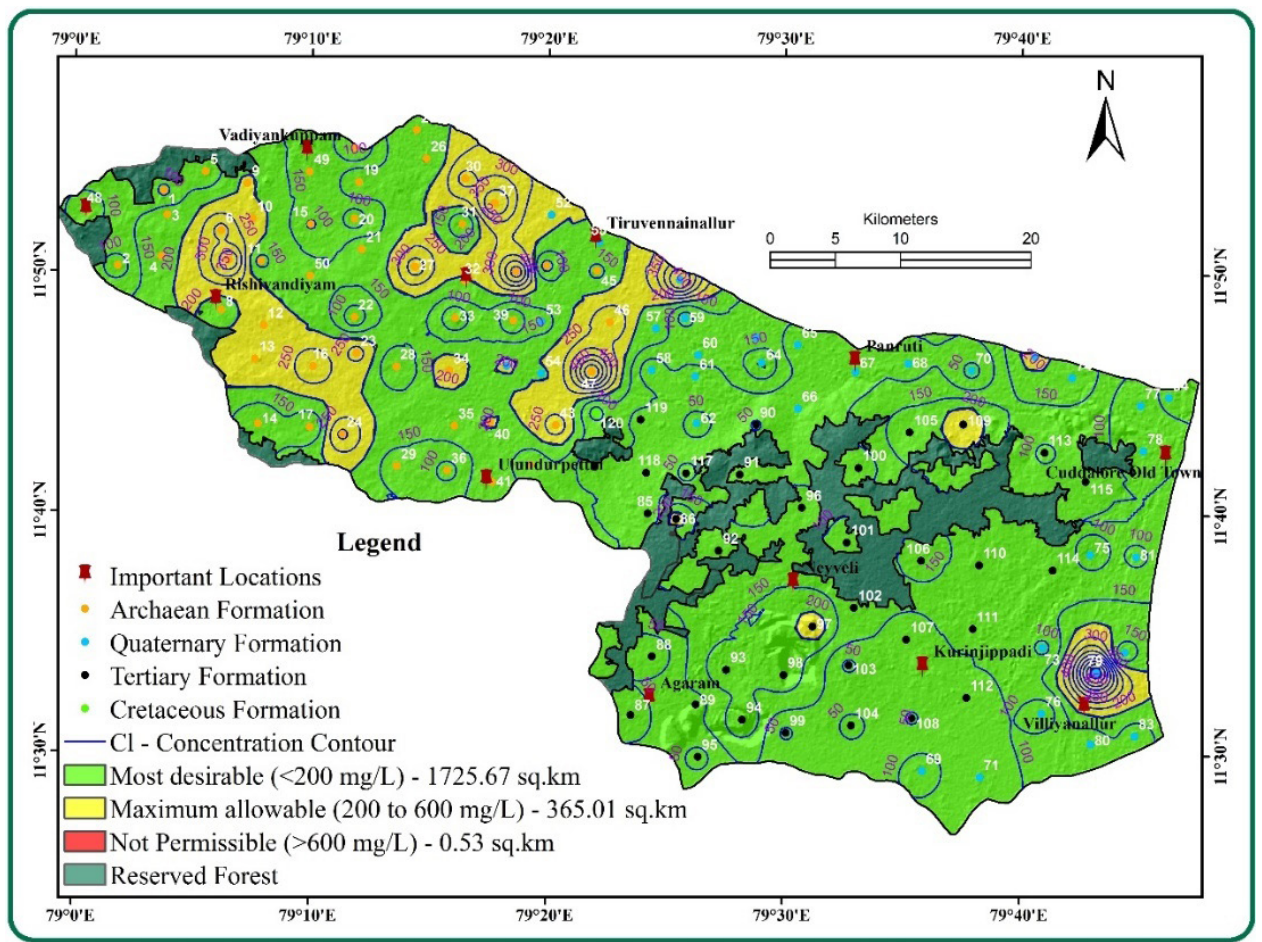

Fig. 6. Chloride spatial variation map - WHO 2017

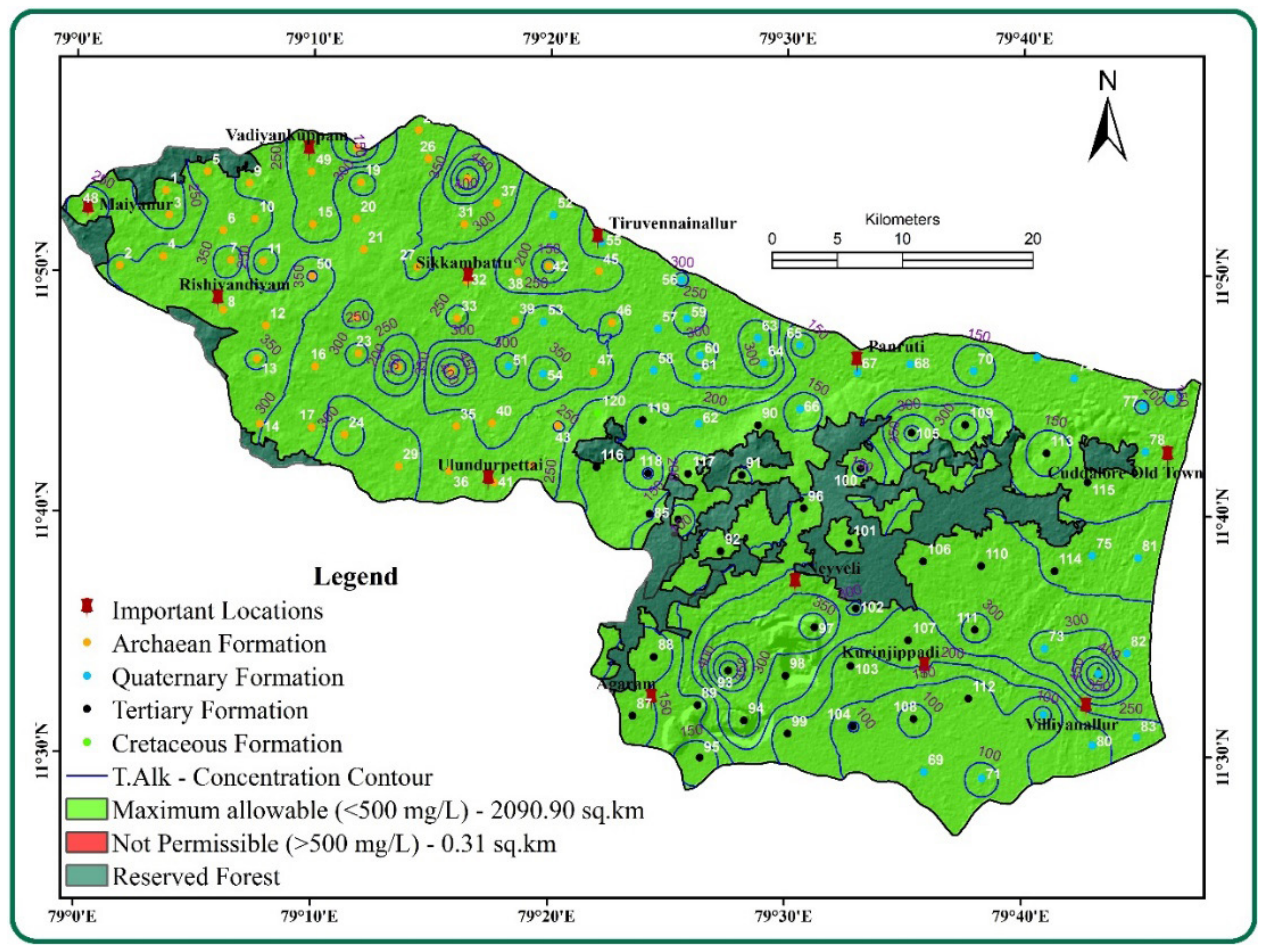

Fig. 7. Total Alkalinity spatial variation map - WHO 2017

that the Quaternary formation has the highest value of $\mathrm{Cl}^{-}(816 \mathrm{mg} / \mathrm{l})$ and T.alk. $(624 \mathrm{mg} / \mathrm{L})$. Chloride $\left(\mathrm{Cl}^{-}\right)$ and Total Alkalinity values are observed to be within the limiting value for WHO 2017 in the Archaean and Tertiary formations during this season.

The higher values of $\mathrm{Cl}$ and Total Alkalinity were found at Periyappattu-79 (632 mg/L and $508 \mathrm{mg} / \mathrm{L})$ station. These sample places were located on the sea shore line. There may be sea water intrusion to the fresh groundwater. The higher values were noticed in only one pocket of the shore line of the Gadilam River Basin. The rest of the section had less than $600 \mathrm{mg} / \mathrm{L}$. The high chloride and Total Alkalinity concentration can be attributed to sea water intrusion (Sameer e al., 2011). 
The EC values are found higher in some samples in all the formations. The EC values of the three formations show that Quaternary formation has the highest value $(3400 \mu \mathrm{mohs} / \mathrm{cm})$ while the maximum value of the Archaean formation is $2600 \mu \mathrm{mohs} / \mathrm{cm}$ and the maximum value of the Tertiary formations is $1887 \mu \mathrm{mohs} / \mathrm{cm}$. This may be due to the presence of concentrated colloids in the water (Verma et al., 2012).
From the spatial variation (Fig. 8) with contour lines of EC map, twenty-three samples in the Archaean formation are seen exceeding the limit for drinking purposes. Some of the sample places are located in between two rock types, many of the sample places are located in $\mathrm{Hbg}$, there may be anthropogenic activities. Therefore, the weaker plane may have significant control on rock water interaction.

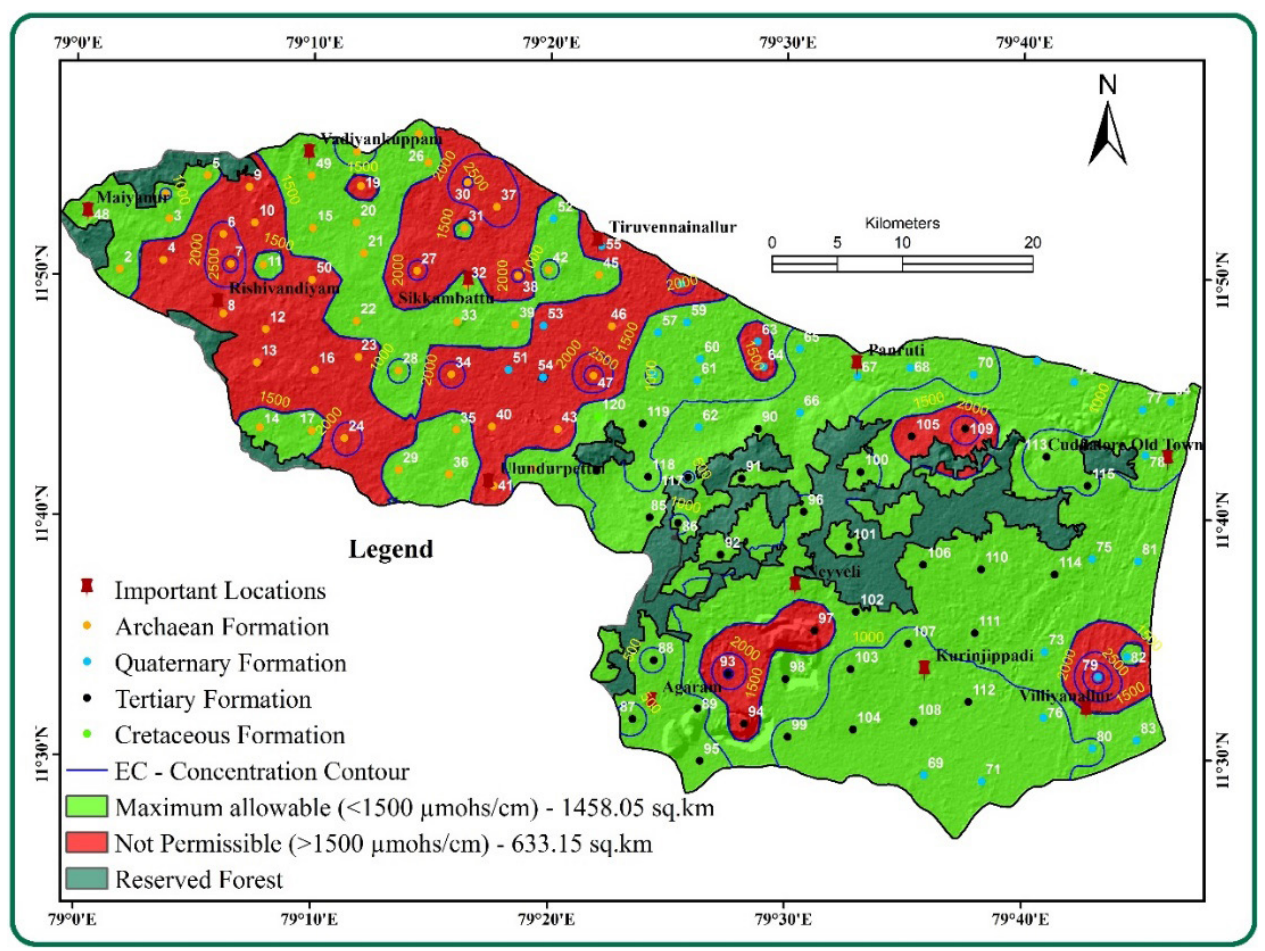

Fig. 8. EC spatial variation map - WHO 2017

Seven samples were studied from the Quaternary formation. These sample places were located in between (contact zone) the flood basin deposit with hornblendebiotite gneiss. Therefore, the weaker plain may have significant influence on rock water interaction.

In five samples from the Tertiary formation groundwater quality was found to be excess of the permissible limit. These sample places were located in between (contact zone) sand stone, clay, lignite deposit with flood basin deposit and the nearby lignite mining area. Therefore, the weaker plane may have major influence on rock water interaction and anthropogenic activities. The higher concentration occurred along the middle of the basin and east coast of the Gadilam River Basin. The rest of the area showed a value of than $1500 \mu \mathrm{mohs} / \mathrm{cm}$.

Total Dissolved Solids (TDS) values were within limiting value for WHO 2017 in Tertiary formations during this season. Some samples in the other two formations were above limiting values $d$ due to the presence of common mineral salts that are dissolved in water (Al Dahaan et al., 2016).

From the spatial variation with contour lines of TDS map (Fig. 9) two samples in the Archaean formation are seen to exceed the limit for drinking purposes,. These sample places were located in hornblende-biotite gneiss with agricultural land and in between (contact zone) hornblende-biotite gneiss and the flood basin. The reason for the high concentration may be that the weaker plain has significant influence on rock water interaction.

Only two samples were studied in the Quaternary formation. These sample places were located in between (contact zone) the flood basin deposit with hornblendebiotite gneiss. Therefore, the weaker plane may have a substantial impact on rock-water interaction.

Spatial Multi-Criteria Evaluation (SMCE) Analysis for overall Groundwater Quality 


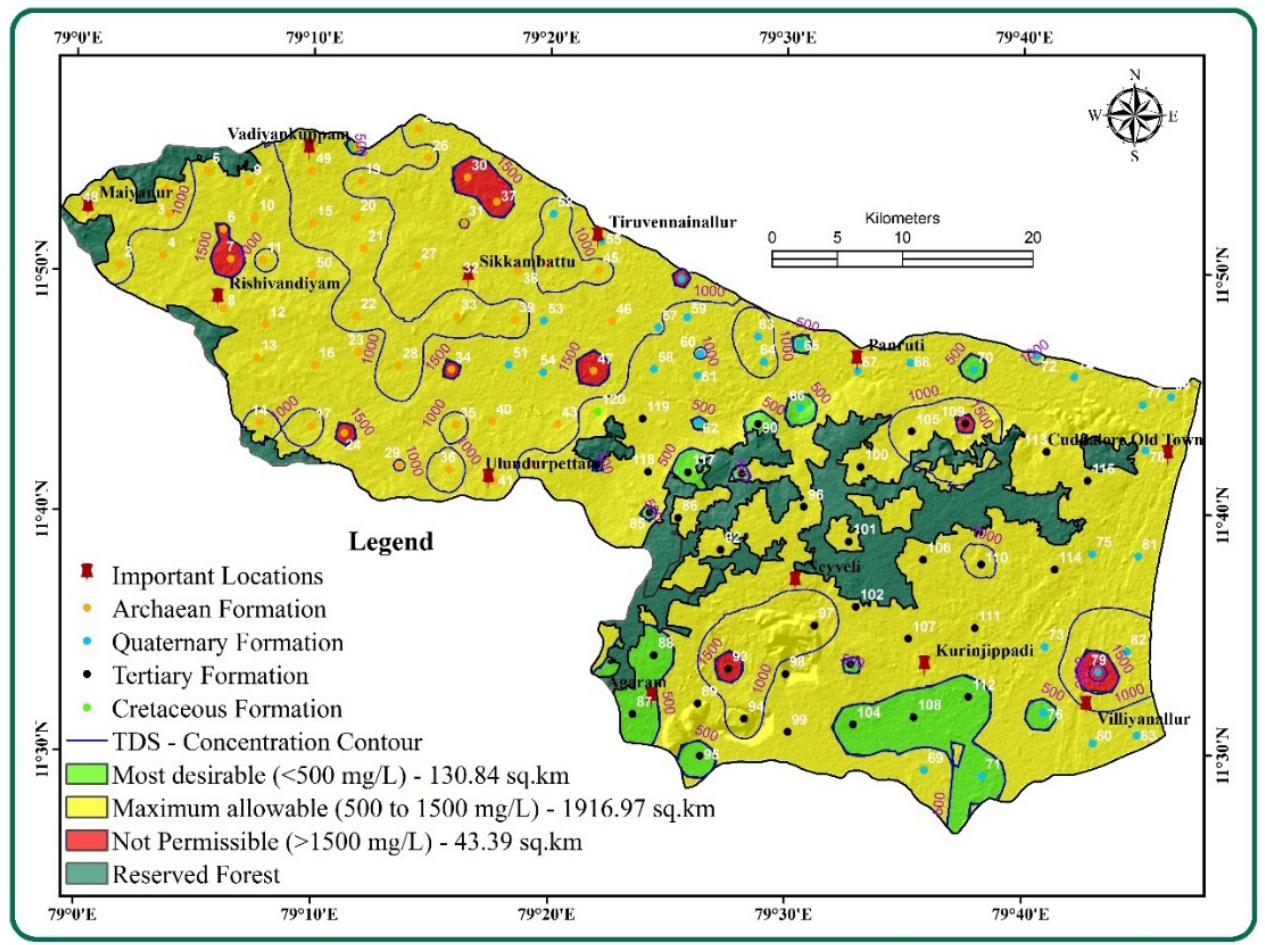

Fig. 9. TDS spatial variation map - WHO 2017

The physico-chemical parameters of contamination have a significant role in human health problems. Major groundwater quality elements were analyzed and their sources and impacts were identified using SMCE method and quality assessment. After creating the entire thematic layers based on the Table 2 Spatial
Multi-Criteria Evaluation Rank of WHO 2017 standard of Groundwater, SMCE overlay analysis was conducted using all the thematic layers and finally, the locations of groundwater quality zones were mapped in the Gadilam River Basin (Fig. 10).

Table 2. Spatial Multi-Criteria Evaluation Rank on Risk Hazardous of Groundwater

\begin{tabular}{|c|c|c|c|c|}
\hline \multirow{2}{*}{$\begin{array}{l}\text { Physio-Chemical } \\
\text { Parameters }\end{array}$} & \multicolumn{2}{|c|}{ Desirable Values for WHO - 2017} & \multirow{2}{*}{$\begin{array}{l}\text { Human health problems } \\
\text { for Present Study }\end{array}$} & \multirow{2}{*}{$\begin{array}{l}\text { Spatial Multi-criteria Evaluation Rank on } \\
\text { human health problems of Groundwater }\end{array}$} \\
\hline & $\begin{array}{l}\text { Most desirable } \\
\text { limits }\end{array}$ & $\begin{array}{c}\text { Maximum } \\
\text { allowable limits }\end{array}$ & & \\
\hline $\mathrm{Ca}^{2+}(\mathrm{mg} / \mathrm{L})$ & 75 & 200 & Above $200 \mathrm{mg} / \mathrm{L}$ & Within permissible limit $=0$ \\
\hline $\mathrm{Mg}^{2+}(\mathrm{mg} / \mathrm{L})$ & 50 & 150 & Above $150 \mathrm{mg} / \mathrm{L}$ & Within permissible limit $=0$ \\
\hline $\mathrm{Na}^{+}(\mathrm{mg} / \mathrm{L})$ & - & 200 & Above $200 \mathrm{mg} / \mathrm{L}$ & 8 \\
\hline $\mathrm{K}^{+}(\mathrm{mg} / \mathrm{L})$ & - & 12 & Above $12 \mathrm{mg} / \mathrm{L}$ & 8 \\
\hline $\mathrm{NO}_{3}{ }^{2-}(\mathrm{mg} / \mathrm{L})$ & - & 45 & Above $45 \mathrm{mg} / \mathrm{L}$ & Within permissible limit $=0$ \\
\hline $\mathrm{Cl}^{-}(\mathrm{mg} / \mathrm{L})$ & 200 & 600 & Above $600 \mathrm{mg} / \mathrm{L}$ & 8 \\
\hline $\mathrm{F}^{-}(\mathrm{mg} / \mathrm{L})$ & - & 1.5 & Above $1.5 \mathrm{mg} / \mathrm{L}$ & Within permissible limit $=0$ \\
\hline $\mathrm{SO}_{4}^{2-}(\mathrm{mg} / \mathrm{L})$ & 200 & 400 & Above $400 \mathrm{mg} / \mathrm{L}$ & Within permissible limit $=0$ \\
\hline $\mathrm{HCO}_{3}^{-}(\mathrm{mg} / \mathrm{L})$ & 300 & 500 & Above $500 \mathrm{mg} / \mathrm{L}$ & Within permissible limit $=0$ \\
\hline $\mathrm{EC}(\mu \mathrm{mohs} / \mathrm{cm})$ & - & 1500 & Above $1500(\mu \mathrm{mohs} / \mathrm{cm}$ & 10 \\
\hline $\mathrm{pH}$ & $6.5-8.5$ & 9.2 & Below 6.5 and Above 8.5 & Within permissible limit $=0$ \\
\hline TDS $(\mathrm{mg} / \mathrm{L})$ & 500 & 1500 & Above $1500 \mathrm{mg} / \mathrm{L}$ & 10 \\
\hline $\mathrm{TH}(\mathrm{mg} / \mathrm{L})$ & 100 & 500 & Above $500 \mathrm{mg} / \mathrm{L}$ & 8 \\
\hline T.Alk (mg/L) & - & 500 & Above $500 \mathrm{mg} / \mathrm{L}$ & 8 \\
\hline & & & Total Rank & $60 / 70$ \\
\hline
\end{tabular}

Three significant factors were extracted by 'Most desirable, 'Maximum allowable' and 'Not permissible' explaining 60 of total variance (Fig. 10). Overall analysis reveals that 19 samples (Archaean-12, Quaternary-4, Tertiary-3) indicate risk of human health problems. 97 groundwater samples (Archaean-38, Quaternary-29 and
Tertiary-29 and Cretaceous-1) indicate moderate risk of human health problem zones. 4 groundwater sites (Archaean-0, Quaternary-1 and Tertiary-3) are classified as No problem zones. 


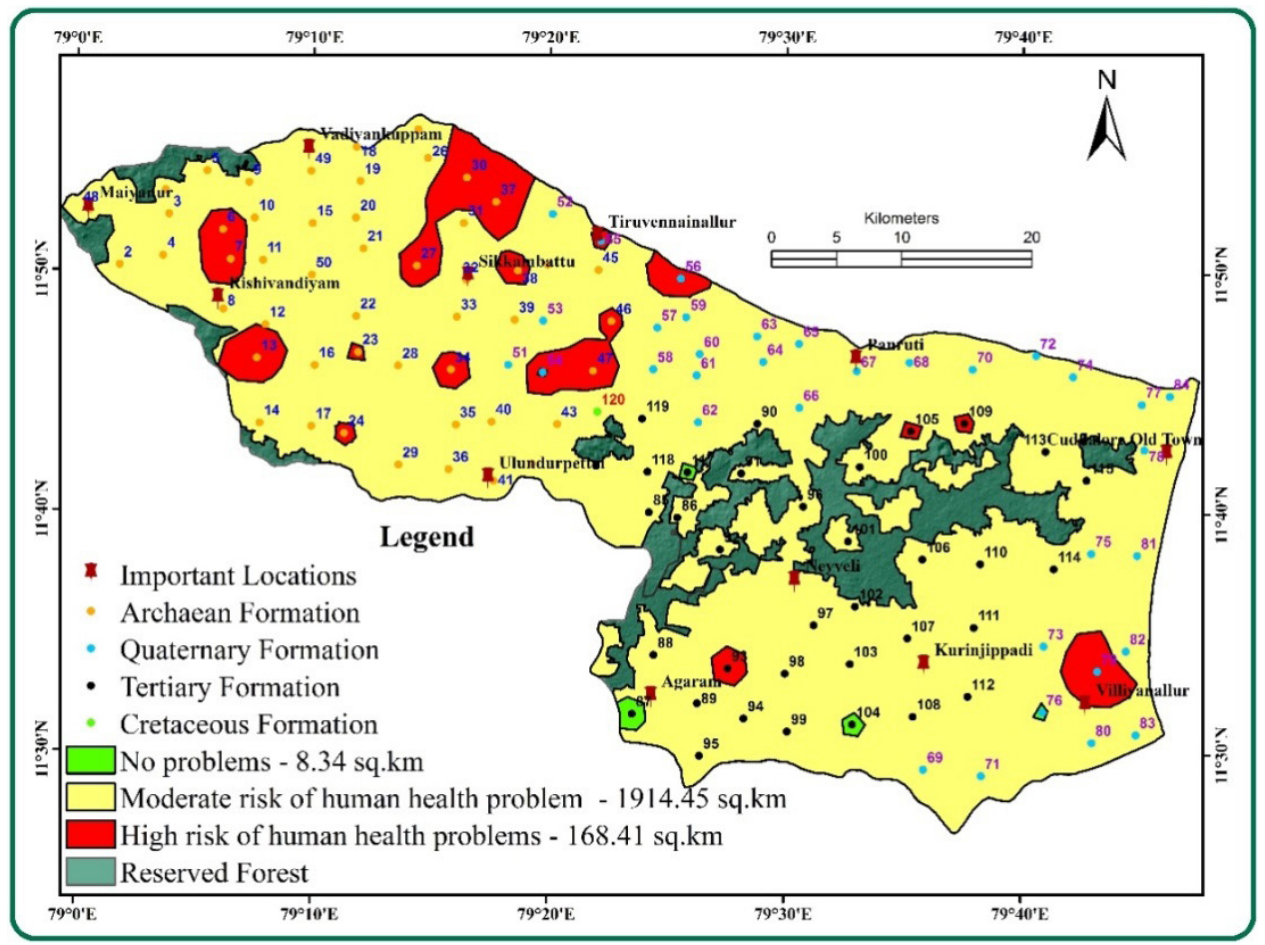

Fig. 10. SMCE Rank on human health problems of Groundwater Map

The 'Not permissible' or risk of human health problems zones were noticed in between (contact zone) hornblende-biotite gneiss and the flood basin and the reason for high concentration in the Archaean formation, it is that the Weaker plane present in the rocks may have significant influence of rock water interaction. Similarly the lineaments' intersection with the river basin and sea shore area in the Quaternary formation especially near SIPCOT industry and lignite mining area are the reason for high concentration in the Tertiary formation (Varol, 2019; Barra-Rocha, et al., 2019; Hou, et al., 2019).

\section{Groundwater Quality Index for Irrigation.}

The water quality index for groundwater samples according to formations is given below. The values of groundwater water quality index demonstrate its appropriateness for irrigation uses. The WQI can classified into five types such as Excellent $(<50)$, Good
(51-100), Poor (101-150), Very poor (151-200) and Worst $(>200)$.

The Archaean formation WQI values are found to be less than 50 in 29 stations, which come under the category "Excellent", and 21 samples fall under the category "Good" category for irrigation uses. In order of WQI, these stations come under the category of "Excellent" and "Good" (Table 3). The Quaternary formation WQI values are observed to be less than 50 in 27 stations out of 34 locations, these stations come under "Excellent" and 7 locations fall under the category of "Good". The Tertiary formation WQI values are found to be less than 50 in 30 stations out of 35 locations, these come under the category "Excellent" and 5 stations fall under the category "Good". Overall 87 stations came under the category of "Excellent" and the stations 33 fell under "Good" in this study period (Table 3).

Table 3. Archaean Formation Water Quality Index

\begin{tabular}{|l|c|c|c|}
\hline \multirow{2}{*}{\multicolumn{1}{|c|}{ Formations }} & \multirow{2}{*}{ WQI Values - June 2019} & Excellent & WQI Classes \\
\cline { 3 - 4 } & & 29 & 21 \\
\hline Archaean formation & 16.49 to 85.46 & 27 & 7 \\
\hline Quaternary formation & 16.34 to 94.97 & 30 & 5 \\
\hline Tertiary formation & 12.44 to 78.54 & & Good \\
\hline
\end{tabular}

From the spatial variation of water quality index map (Fig. 11) classifications of groundwater for irrigation purposes are seen. An enormous area comes under the category "Excellent". Some large patches studied in the upper part and small spots noticed in lower part of the study area come under the category "Good" for irrigation purposes. The 'Good' zones were noticed in between (contact zone) hornblende-biotite gneiss and the flood basin and the reason for high concentration in the Archaean formation, is that the Weaker plane may 


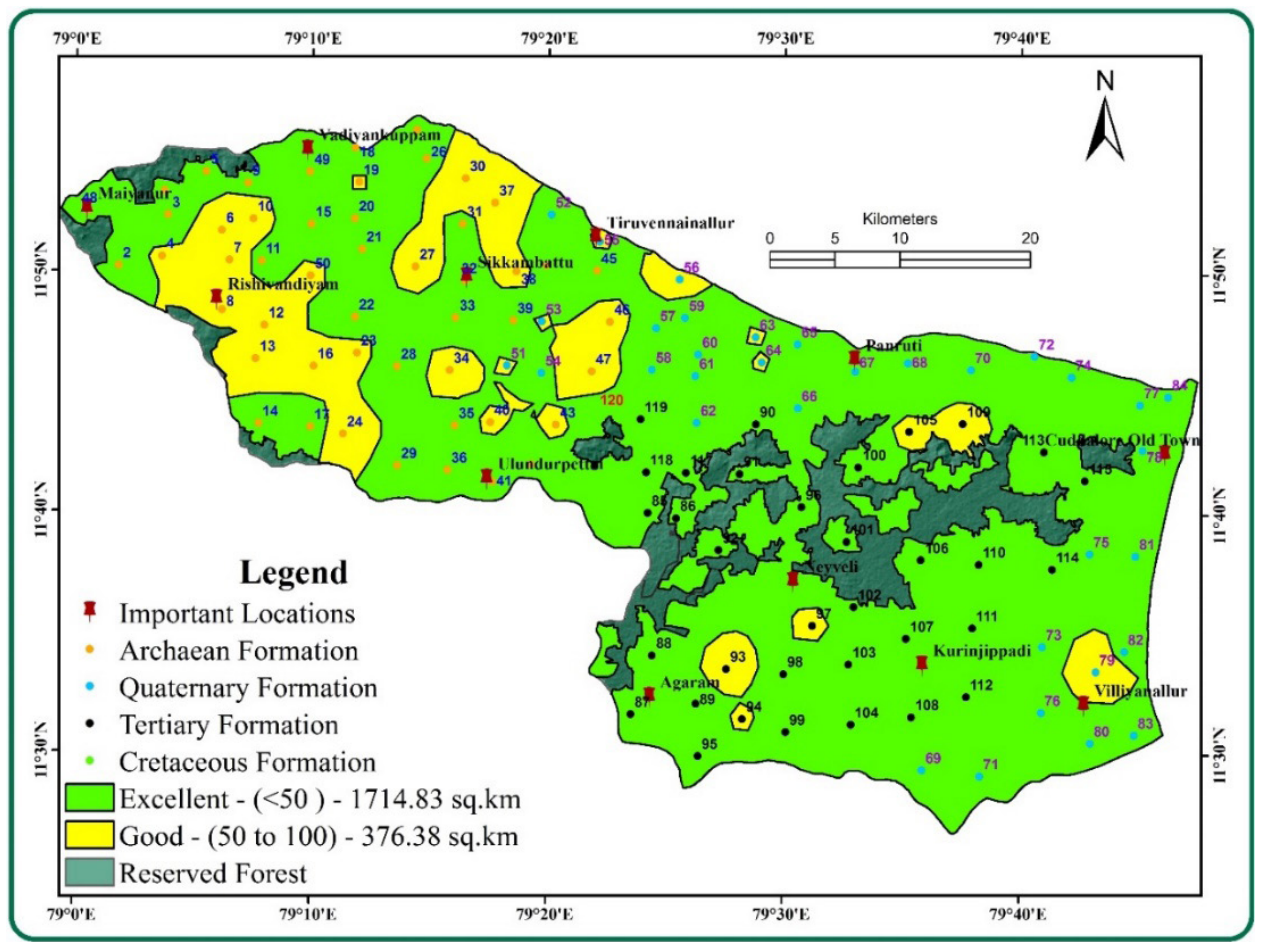

Fig. 11. Spatial variation of water quality index map

be affected by the significant influence of rock water interaction, lineaments intersection with the river and sea shore area in the Quaternary formation and by the nearby SIPCOT industry and Lignite mining area in the Tertiary formation.

\section{Piper Trilinear Diagrams}

The geochemical evaluation can be understood by six classes such as Calcium-Bicarbonate, SodiumChloride, Calcium-Sodium-Bicarbonate, CalciumMagnesium-Chloride, Calcium-Chloride and Sodium-
Bicarbonate types. It clearly explains the different categories of dominant cations and anions in various formations.

In the Archaean formation piper trilinear diagrams (Figures 12 and Table 4) it is observed that the majority of samples come under the No dominant type in the cation and anion triangles. A further $10 \%$ of the stations fall under the $\mathrm{NaCl}$ type and rest of the stations fall under the mixed $\mathrm{CaMgCl}$ type. This may be due to the rock water interaction of crystalline nature of rocks and anthropogenic activities (Veena Srinivasan et al., 2014).

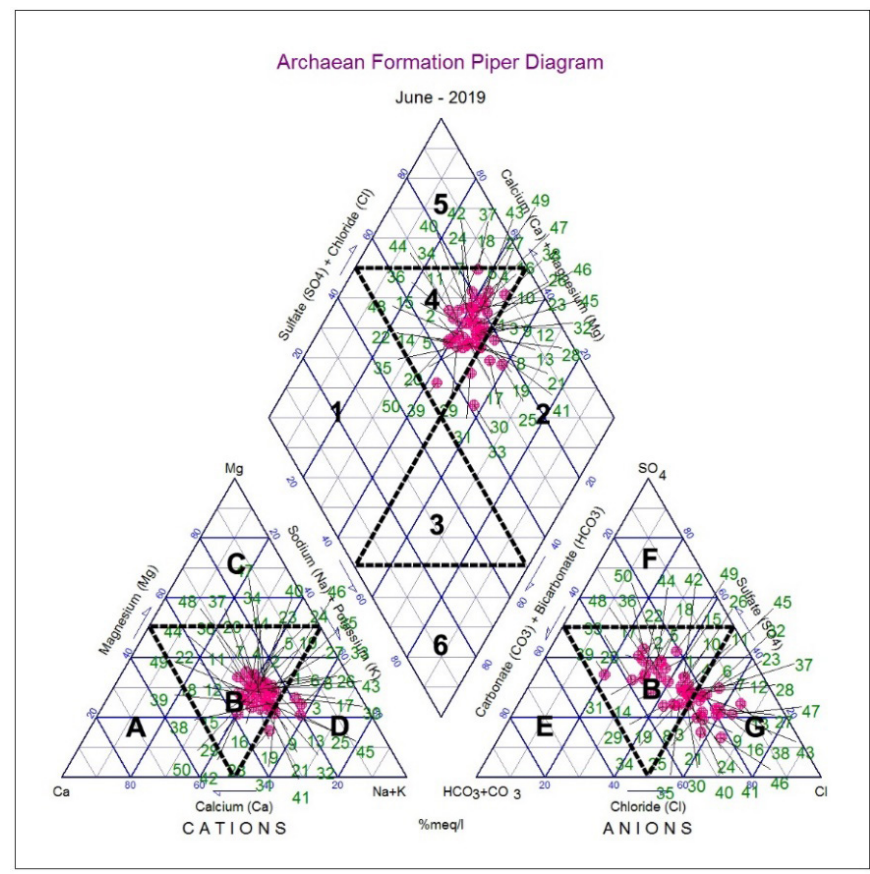

Fig. 12. Piper Trilinear Diagrams - Archaean Formation 
Table 4 Piper Trilinear diagram

\begin{tabular}{|c|c|c|c|c|c|}
\hline \multirow{2}{*}{$\begin{array}{l}\text { Geological } \\
\text { Formations }\end{array}$} & \multirow{2}{*}{ Class No. } & \multirow{2}{*}{ Class } & \multicolumn{3}{|c|}{ June - 2019} \\
\hline & & & Stations & No. of Samples & Percentage of samples \\
\hline \multirow{6}{*}{$\begin{array}{l}\text { Archaean } \\
50 \text { Samples }\end{array}$} & 1 & $\mathrm{CaHCO}_{3}$ & Nil & Nil & Nil \\
\hline & 2 & $\mathrm{NaCl}$ & $25,28,30,31,33$ & 5 & $10 \%$ \\
\hline & 3 & Mixed $\mathrm{CaNaHCO}_{3}$ & Nil & Nil & Nil \\
\hline & 4 & Mixed $\mathrm{CaMgCl}$ & All samples except 5 samples & 45 & $90 \%$ \\
\hline & 5 & $\mathrm{CaCl}$ & Nil & Nil & Nil \\
\hline & 6 & $\mathrm{NaHCO}_{3}$ & Nil & Nil & Nil \\
\hline \multirow{6}{*}{$\begin{array}{l}\text { Quaternary } \\
34 \text { Samples }\end{array}$} & 1 & $\mathrm{CaHCO}_{3}$ & Nil & Nil & Nil \\
\hline & 2 & $\mathrm{NaCl}$ & $54,55,56,57,58,60,68,70,73,79,83$ & 11 & $32 \%$ \\
\hline & 3 & Mixed $\mathrm{CaNaHCO}_{3}$ & Nil & Nil & Nil \\
\hline & 4 & Mixed $\mathrm{CaMgCl}$ & All samples except 11 samples & 23 & $68 \%$ \\
\hline & 5 & $\mathrm{CaCl}$ & Nil & Nil & Nil \\
\hline & 6 & $\mathrm{NaHCO}_{3}$ & Nil & Nil & Nil \\
\hline \multirow{6}{*}{$\begin{array}{c}\text { Tertiary } \\
35 \text { Samples }\end{array}$} & 1 & $\mathrm{CaHCO}_{3}$ & $\begin{array}{r}\text { Nil } \\
\end{array}$ & Nil & Nil \\
\hline & 2 & $\mathrm{NaCl}$ & $86,89,90,102,106,111$ & 6 & $17 \%$ \\
\hline & 3 & Mixed $\mathrm{CaNaHCO}_{3}$ & $\begin{array}{l}\text { Nil } \\
\end{array}$ & Nil & Nil \\
\hline & 4 & Mixed $\mathrm{CaMgCl}$ & All samples except 6 samples & 29 & $83 \%$ \\
\hline & 5 & $\mathrm{CaCl}$ & Nil & Nil & Nil \\
\hline & 6 & $\mathrm{NaHCO}_{3}$ & Nil & Nil & Nil \\
\hline $\begin{array}{c}\text { Cretaceous } \\
1 \text { Sample }\end{array}$ & 4 & Mixed $\mathrm{CaMgCl}$ & 120 & 1 & $100 \%$ \\
\hline
\end{tabular}

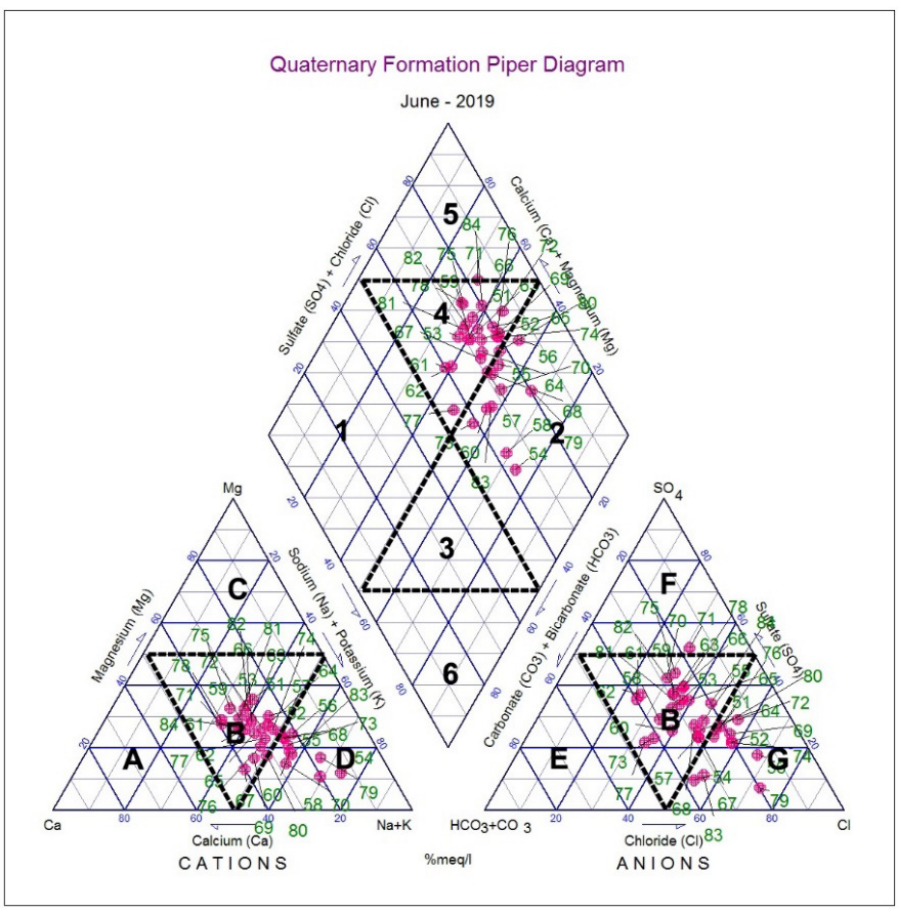

Fig. 13. Piper Trilinear Diagrams - Quaternary Formation

The Quaternary formation plots (Figures 13 and Table 4) reveals that the $32 \%$ of the stations fall under the Sodium-Potassium type in the cation triangle and Chloride type in the anion triangle. $68 \%$ of the stations fall under the mixed $\mathrm{CaMgCl}$ type. This may be due to sea water intrusion and anthropogenic activities (Vikas Tomar et al., 2012).
The Tertiary formation piper trilinear diagrams (Figures 14 and Table 4) results show that the majority of samples (more than $94 \%$ ) come under the No dominant type in the cation and anion triangles. The following stations $86,89,90,102,106,111$ fall under the $\mathrm{NaCl}$ type and rest of the stations fall under the mixed $\mathrm{CaMgCl}$ type. This may be due to the leaching of alkali salts (Umapathy et al., 2011). 


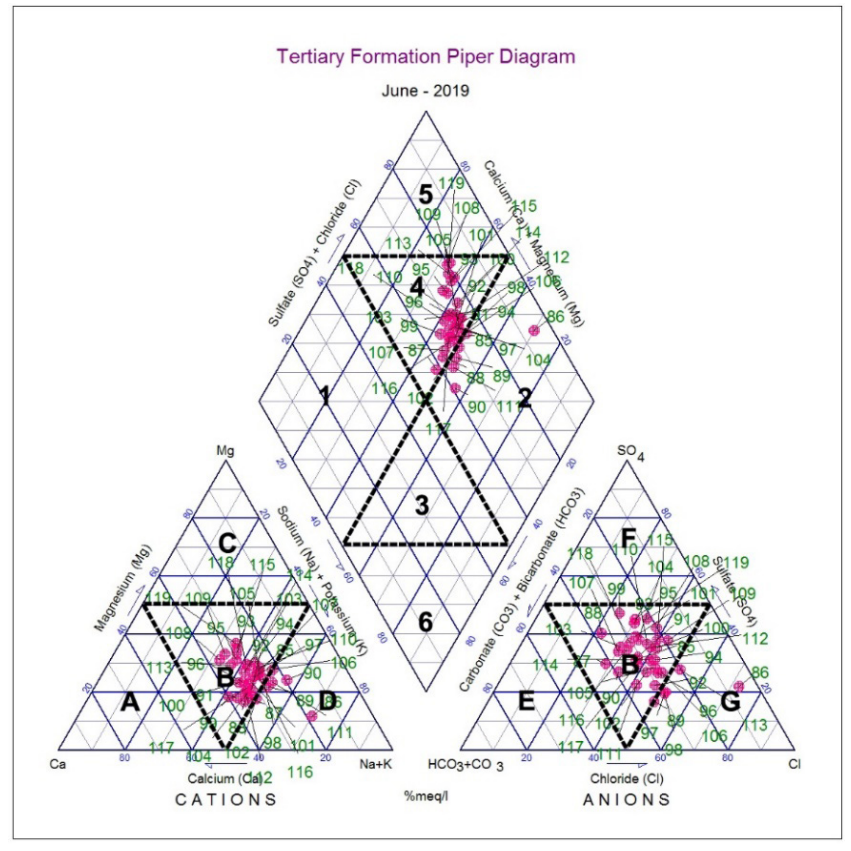

Fig. 14. Piper Trilinear Diagrams - Tertiary Formation

The Cretaceous formation piper trilinear diagrams and anion triangles. The following stations fall under (Figures 15 and Table 4) results show that the studied the mixed CaMgCl type. This may be due to the rock samples come under the No dominant type in the cation water interaction and anthropogenic activities.

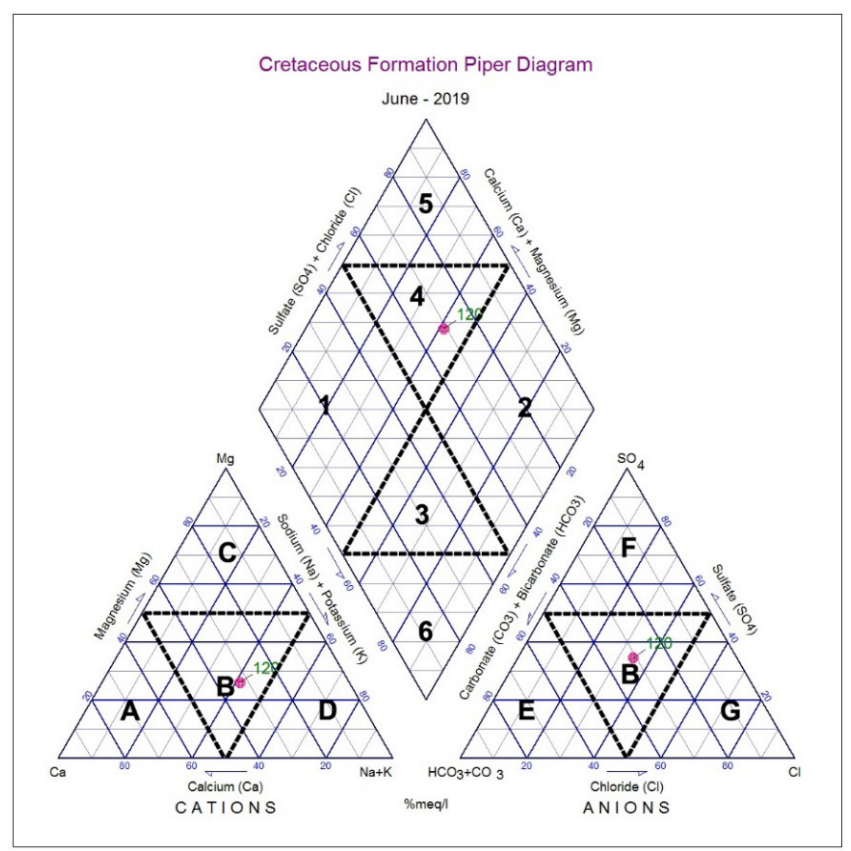

Fig. 15. Piper Trilinear Diagrams - Cretaceous Formation

Hence, this study indicates that the Piper trilinear classification of groundwater samples fall in the field of mixed $\mathrm{Ca}-\mathrm{Mg}-\mathrm{Cl}$, and No dominance, some of the samples in $\mathrm{Na}-\mathrm{K}, \mathrm{Cl}$ types of water. So based on the Piper trilinear, the groundwater samples are fit for drinking and irrigation purposes for all formations.

\section{Conclusions.}

The highest values of the Quaternary formation physio-chemical parameters are noticed in the rainy season due to the confined aquifer (Neyveli Aquifer) containing fertilizer used in increasing agricultural activities. The groundwater quality parameters such as Calcium $\left(\mathrm{Ca}^{2+}\right)$, Magnesium $\left(\mathrm{Mg}^{2+}\right)$, Nitrate $\left(\mathrm{NO}_{3}{ }^{2-}\right)$, 
Fluoride ( $\left.\mathrm{F}^{-}\right)$, Sulphate $\left(\mathrm{SO}_{4}^{2-}\right)$, Bi-carbonate $\left(\mathrm{HCO}_{3}^{-}\right)$ and Percentage of Hydrogen $(\mathrm{pH})$ values are observed to be within limiting value for WHO 2017 in all the formations during this season.

The EC and TDS values are in excess of the permissible limit for some stations in all the formations. The TH and T. Alk.values are seen as exceeding the limit for drinking purposes among 11 samples in Archaean formation and in 2 samples in Quaternary formation.

$\mathrm{K}$ values are seen to exceed the limit for drinking purposes in $96 \%$ of the samples of the Archaean formation, $74 \%$ of the samples of Quaternary formation $94 \%$ of the samples of the tertiary formation. The $\mathrm{Cl}$, $\mathrm{NO}_{3}$ values are exceeding the limit for drinking purposes in only two samples of Quaternary formation whereas all the samples of Archaean and Tertiary formations are within the limit.

The Water Quality Index demonstrates the appropriateness of the water for irrigation uses. The

\section{References}

Akhilesh Jinwal., Savita Dixit., 2008. Pre and postmonsoon variation in physicochemical characteristics in groundwater quality of Bhopal "The city of lakes" India. Asian J. Exp. Sci., 22(3): 311-316.

Al Dahaan, S.A., Nadhir Al-Ansari, M.A., Sven Knutsson., 2016. Influence of groundwater hypothetical salts on electrical conductivity, total dissolved solids. Engineering., 8 (11): 823-830.

Ambrina Sardar Khan., Prateek Srivastava., 2012. Physicochemical characteristics of groundwater in and around Allahabad city: A statistical approach. Besr.Org.In., 1(2): 28-32.

Asghari, F.B., Mohammadi, A.A., Dehghani, M.H., Yousefi, M.,2018. Data on assessment of groundwater quality with application of ArcGIS in Zanjan, Iran. Data Brief $18: 375$

Bagade, S.P., 1995. unpublished Report, Groundwater potential and causes of salinity in Vasai-Virar area, Thane district, Maharastra, Central Groundwater Board, Nagpur, 12.

Barra-Rocha., C.H., Fernandes-Costa., H., Pimenta-Azevedo, L.,2019. Heavy metals in the São Mateus Stream Basin, Peixe River Basin, Paraiba do Sul River Basin, Brazil. Ambient Agua Interdiscip. J. Appl. Sci. 14, 1-13.

Brindha, K., Kavitha, R., 2014.Hydrochemical assessment of surface water and groundwater quality along Uyyakondan channel, south India Environ Earth Sci 73 5383-5393 doi:10.1007/s12665-014-3793-5

Brindha, K., Vaman, K.V.N., Srinivasan, K., Babu, M.S., Elango, L., 2013. Identification of surface watergroundwater interaction by hydrogeochemical indicators and assessing its suitability for drinking and irrigational purposes in Chennai, Southern India Appl Water Sci 4 159-174 doi: 10.1007/s13201-013-0138-6

Duraiswami, R.A., Thigale, S.S., Pawar, N.J., Meshram, D.C., 2000. Coastal environment of Maharastra: issues
WQI values for the Archaean, Quaternary and Tertiary formations are found to be less than $100 \mathrm{meq} / \mathrm{L}$ in all stations. In order of WQI, these stations come under the category of "Excellent" and "Good".

The Piper trilinear classification of groundwater samples fall in the field of mixed $\mathrm{Ca}-\mathrm{Mg}-\mathrm{Cl}$, and $\mathrm{No}$ dominance, some of the samples represent $\mathrm{Na}-\mathrm{K}, \mathrm{Cl}$ types of water. So based on the Piper trilinear, the groundwater samples are fit for drinking and irrigation purposes for all formations.

\section{Acknowledgements.}

The authors acknowledge the Survey of India and Geological Survey of India for providing topographical maps and Geology map respectively. The opinions expressed in this paper are those of the authors and many of the facilities are provided Department of Geology, Government Arts College (Auto), Salem.

vis-à-vis development, contributions to Environmental Geoscience, Arawali Publ. New Delhi, 59-73.

American Public Health Association., 2005. Standard methods for the examination of water and wastewater. American Public Health Association (APHA): Washington, DC, USA.

Horton, R.K., 1965. An index number system for rating water quality. Journal of Water Pollution Control Federation, 37(3), 300-306.

Hou, S., Zheng, N., Tang, L., Ji, X., Li, Y., Hua, X.,2019. Pollution characteristics, sources, and health risk assessment of human exposure to $\mathrm{Cu}, \mathrm{Zn}, \mathrm{Cd}$ and $\mathrm{Pb}$ pollution in urban street dust across China between 2009 and 2018. Environ. Int. 128, 430-437.

Kumar, S.K., Logeshkumaran, A., Magesh, N.S., Godson, P.S., Chandrasekar, N., 2014. Hydro-geochemistry and application of water quality index (WQI) for groundwater quality assessment, Anna Nagar, part of Chennai City, Tamil Nadu, India Appl Water Sci 5335-343 doi: 10.1007/s13201-014-0196-4

Landwehr, J.M., Deininger, R.A., Harkins, R.D., 1974. An objective water quality index. Journal of Water Pollution Control Federation, 1804-1809.

Layeek Ahamed, R., Krishna kumar, S., Manikandan, A., Rajaprabhu, P., Selvarasu, R., 2014. Appraisal of water quality and hydrogeochemistry of surface and groundwater in Neyveli region, Tamil Nadu, India. Enviro Geo ChimicaActa (2014) 1(1):67-76

Mahmoud, S., Shahub Mahmoud, S., Ibrahim Maie, I., Algammal Mohamed., Abdelgalil Moktar, S., Alatrash., 2016. Seasonal analysis of physico-chemical parameters of ground and surface water in Kaam area, Libya. JESTFT. 10(6): 46-50.

Mishra, P.C., Patel, R.K., 2001. Study of the pollution load in the drinking water of Rairangpur, a small tribal 
dominated town of North Orissa. Indian J Environ Ecoplan, 5(2), 293-298.

Muthusaravanan, S., Sivarajasekar, N., Vivek, J.S., Paramasivan, T., Naushad, M., Prakashmaran, J., Gayathri, V., Al-Duaij, O.K., 2018. Phytoremediation of heavy metals: mechanisms, methods and enhancements. Environ ChemLett 16:1339-1359

Naik, S., Purohit, K.M., 2001. Studies on water quality of river Brahmani in Sundargarh district, Orissa. Indian J Environ Ecoplan, 5(2), 397-402.

Pirsaheb, M., Fazlzadehdavil, M., Hazrati, S., Sharafi, K., Khodadadi, T., Safari, Y.,2014. A survey on nitrogen and phosphor compounds variation process in wastewater stabilization ponds. Pol J Environ Stud 23:831-834

Prasanna, M.V., Chidambaram, S., Hameed, A.S., Srinivasamoorthy, K., 2011.Hydrogeochemical analysis and evaluation of groundwater quality in the Gadilam river basin, Tamil Nadu, India J Earth SystSci 120 85-98 Indian Academy of Sciences

Rajesh, R., Brindha, K., Elango, L.,2015.Groundwater Quality and its Hydrochemical Characteristics in a Shallow Weathered Rock Aquifer of Southern India Water Qual Expo Health 7 515-524 doi: 10.1007/ s12403-015-0166-6

Rajmohan, N., Elango, L., Ramachandran, S., Natarajan, M., 2000. Major ion correlation in groundwater of Kancheepuram region, south India: Ind. J. Environ. Protection., 20(3): 188-193.

Rina, K., Dutta, P.S., Mukherjee, S., 2011. Characterization and Evaluation of processes governing the groundwater quality in parts of the Sabarmati basin, Gujarat using hydrochemistry integrated with GIS Hydrological Processes 26 1538-1551 doi: 10.1002/hyp.8284

Sameer, V., Yamakanamardi Hampannavar, U.S., Purandara, B.K., 2011. Assessment of chloride concentration in groundwater: A case study for Belgaum city. Int. J. Environ. Sci., 2 (1): 271-280.

Sanjay, Dahasahasra, V., 2007. 'A model for transforming an intermittent water supply system', J. Geosp., Vol. 6-6, 34-39.

Shankar, K., Aravindan S., Rajendran, S., 2011. Hydrochemical profile for assessing the groundwater quality of Paravanar river sub-basin, Cuddalore District, Tamil Nadu, India. E-Journal of Chemistry, 8(2), 835845.

Singh, C.K., Rina, K., Mallick, J., Singh, R.P., Singh, N., Shashtri, S., Mukherjee, S.,2012a.Chemometric and GIS based analysis of geogenic augmentation of fluoride in groundwater of arid region of India International Journal of Environmental Protection 2, 24-29

Singh, C.K., Shashtri, S.N., Kumari, R., Mukherjee, S., 2012b.Chemometric analysis to infer hydro-geochemical processes in a semi-arid region of India Arabian Journal of Geosciences doi: 10.1007/s12517-012-0597-3

Singh, D.F., 1992. Studies on the water quality index of some major rivers of Pune, Maharashtra. In ProcAcad Environ Biol, 1(1), 61-66.
Sonkamble, S., Sahya, A., Mondal, N.C., Harikumar, P., 2012. Appraisal and evolution of hydrochemical processes from proximity basalt and granite areas of Deccan Volcanic Province (DVP) in India Journal of Hydrology 438-439181-193 doi.org/10.1016/j.jhydrol.2012.03.022

Suresh, M., Gurugnanam, B., Vasudevan, S., Dharanirajan, K., Jawahar Raj, N., 2010. Drinking and Irrigational Feasibility of Groundwater, GIS Spatial Mapping in Upper Thirumanimuthar Sub-basin, Cauvery River, Tamil Nadu. Journal Geological Society of India, 75, March, 518-526.

Thigale, S.S., Pawar, N.J., Meshram, D.C., unpublished, D.S.T., 1998a. Project Report, Hydrogeological and geochemical studies on saline and fresh water interaction in the coastal aquifers, Maharastra, Department of Geology, University of Pune, 82.

Tiwari, T.N., Nayak, S., 2002. Water Quality Index for the Groundwater of Sambalpur Town. Environmental Pollution Research. New Delhi: APH Pub. Corp, 971.

Umapathy, S.,2011. A study on groundwater quality of Neyveli area, Cuddalore district. Tamil Nadu, Int. J. of Geomatics and Geosciences., 2(1):49-56.

Varol, M., 2019. Arsenic and trace metals in a large reservoir: Seasonal and spatial variations, source identification and risk assessment for both residential and recreational users. Chemosphere, 228, 1-8.

Veena Srinivasan., Suresh Kumar, D.,2014. Water management in the Noyyal River Basin A situation analysis, ATREE., 1(1): 1-48.

Verma, O.P., Bushra Khanan., Shruti Shukla., 2012. Determination of physicochemical characteristics of four canals of Allahabad region and its suitability for irrigation. Adv. Appl. Sci. Res., 3(3): 1531-1537.

Vetrimurugan, E., Brindha, K., Elango, L., Osman Muzi Ndwandwe., 2017.Human exposure risk to heavy metals through groundwater used for drinking in an intensively irrigated river delta. Appl Water Sci (2017) 7:3267-3280. DOI 10.1007/s13201-016-0472-6

Vikas Tomar, Kamra, S.K., Kumar, S., Kumar, A., Vishal Khajuria., 2012. Hydrochemical analysis and evaluation of groundwater quality for irrigation in Karnal district of Haryana state, India. Int. J. Environ.Sci., 3(2):756-766.

World Health Organization., 2017. Guidelines for drinkingwater quality. Fourth edition Incorporating the first addendum, 1-631

Wu, Q., Zhao, C., Zhang, Y., 2010. Landscape river water quality assessment by nemerow pollution index. International Conference, IEEE. In Mechanic Automation and Control Engineering (MACE), 2117 2120

Yousefi, M., Dehghani, M.H., Nasab, S.M., Taghavimanesh, V., Nazmara, S., Mohammadi, A.A.,2018. Data on trend changes of drinking groundwater resources quality: a case study in Abhar. Data Brief 17:424-430. 